\title{
Rugged Packaging for Damage Resistant Inertial Fusion Energy Optics
}

Phase I SBIR Final Report

US Department of Energy

DoE Grant DE-FG02-02ER83441

November, 2003

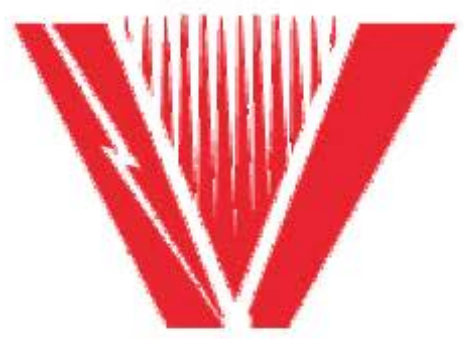

PVD Products, Inc.

231 Andover Street, Wilmington, MA 01887 Larry Stelmack, Principal Investigator 
Disclaimer: This report was prepared as an account of work sponsored by an agency of the United States Government. Neither the United States Government nor any agency thereof, nor any of their employees, makes any warranty, express or implied, or assumes any legal liability or responsibility for the accuracy, completeness, or any third party's use or the results of such use of any information, apparatus, product, or process disclosed, or represents that its use would not infringe privately owned rights.

Reference herein to any specific commercial product, process, or service by trade name, trademark, manufacturer, or otherwise, does not necessarily constitute or imply its endorsement, recommendation, or favoring by the United States government or any agency thereof or its contractors or subcontractors. The views, opinions, findings, recommendations, and conclusions expressed herein are those of the author, and do not necessarily state or reflect those of the United States Government or the Department of Energy.

Acknowledgement: This material is based upon work supported in part by the US Department of Energy, under Phase I SBIR Award number DE-FG02-02ER83441.

\section{Table of Contents}

$\underline{\text { Page }}$

Table of Contents

List of Figures

2

Acknowledgements

Introduction

Program Summary

Background

Experimental Results

Phase II Deposition System Concept

Applications

Conclusions and Recommendations

References 


\section{$\underline{\text { List of Figures }}$}

$\underline{\text { Figure }}$

Page

1. IFE Final Optics Schematic 8

2. Concept for Full-Scale IFE Grazing Incidence Metal Mirror (GIMM) 8

3. Hazards of the IFE Environment 9

4. Negative Effects of IFE Environment on Final Optics 9

5. Properties of an Ideal IFE Final Optics Packaging Coating 10

6. Undesirable Properties of IFE Optical and Protective Coatings 11

7. Contamination Effects on Laser Optics 13

8. Elements of Contamination Control Program 14

9. Properties of Fluorinated Diamondlike Carbon (F-DLC) Coatings 15

10. Fluorocarbon Polymer Structure 16

11. Change in the Contact Angle of Water by Adding Different Elements 16 to DLC Coatings

12. Electrical Conductivity of Ion Beam Deposited Amorphous C:F Films 17 as a Function of Fluorine Concentration

13. Military Specifications for Environmental Durability of Coated Optics 18

14. Deposition of Diamondlike Carbon (DLC) by Ion Beam Assisted 20 Evaporation of Liquid Hydrocarbon Precursors

15. Beneficial Effects of Ion Bombardment on Film Quality 20

16. Illustration of Plasma Immersion Ion Processing (PIIP) Process 21

17. Steps in PIIP Deposition Process 22

18. Depiction of Plasma Immersion Ion Processing 23

19. Exterior View of PIIP System at Southwest Research Institute 24

20. Interior View of SwRI PIIP System during Deposition 24

21. PVD Products Deposition Systems 27

22. PVD Products Reel-to-Reel Tape Coating System 28

23. Phase I Experimental Deposition System Design 29

24. Phase I System Features 3 
25. Phase I System Under Construction 31

26. Components of Heated Source for Evaporation of Liquid Coating 31 Precursors

27. Kaufman-type Ion Source and Mounting Flange (Top View) 32

28. Kaufman-type Ion source and Mounting Flange (Side View) 33

29. Substrate Platform and Rotary Drive Mechanism 34

30. Bottom View of Substrate Platform 35

31. Summary of DLC Coating Test Data 36

32. UV Transmission of Diamondlike Carbon (DLC) Coating 37

33. UV Transmission of Silicated DLC Coating 38

34. UV Transmission of Fluorinated DLC Coating 39

35. Multi-source Magnetron Sputter Deposition System 41

36. End-Hall Ion Source 42

37. Phase II Deposition System Design Concept 45

38. Phase II Deposition System Features 46

39. Phase II Deposition System Design - Interior View 47

40. Phase II Deposition System Design - Rear View 48

41. Phase II Deposition System Design - Top View on 6" Grid 49

42. Potential Government and Commercial Applications 56

43. Optical Surface Testing at OPCO Laboratory 66

44. Laser Damage Test Facility at UC San Diego 67 


\section{$\underline{\text { Acknowledgements }}$}

This project was facilitated by assistance from and helpful discussions with the following people:

- Joseph Murphy, Kim Senay, Michael Paradise, Brendan Howard, Dave Lations, and James Greer, PVD Products, Inc.

- Mark Tillack, University of California at San Diego

- Gene Nardella, US Department of Energy

- John Sethian, Naval Research Laboratory

- James Hirvonen, Army Research Laboratory

- Roger Ramsey and Lewis Desandre, Air Force Research Laboratory

- James Arps and Rong-Hua Wei, Southwest Research Institute

- Mark Dykstra, Susan Bates, and Dan Jasinski, Optical Coating Corporation

- Steve Payne, Lawrence Livermore National Laboratory

- David Reicher, S-Systems Corporation

- Russell Smilgys, Science Applications, Inc.

- Harold Kaufman, Kaufman and Robinson, Inc.

- David Maldari, OPCO Laboratory, Inc. 


\section{$\underline{\text { Introduction }}$}

This document is the Final Technical Report produced by PVD Products, Inc. describing program activities performed under SBIR Phase I Grant number DE-FG0202ER83441, entitled "Rugged Packaging for Damage Resistant Inertial Fusion Energy Optics". The main objective of this program was to assist in determining the best feasible near- term solution to the urgent problem of maximizing the lifetime of the final optical elements on laser fusion reaction chambers. PVD Products evaluated the potentially advantageous new technique of applying transparent diamondlike carbon (DLC) optical packaging coatings to critical IFE high-energy laser (HEL) optical components.

Program Summary. The overall goal of the program is the development and implementation of rugged, low-maintenance protective coatings for costly, contaminationsensitive high-energy Inertial Fusion Energy (IFE) laser optical components, particularly the critical "final optics" required for steering and focusing high-energy ultraviolet laser beams onto inertial confinement fusion targets. The general requirements for IFE optics include very high optical efficiency, smoothness, low distortion, stability, ruggedness, and durability. Their optical and protective coatings must be robust, with no tendency to separate from the substrate.

This program addressed the definition of requirements for IFE optics protective coatings, the conceptual design of the required deposition equipment according to accepted contamination control principles, and the deposition and evaluation of diamondlike carbon test coatings. The program emphasized the importance of contamination control, and the evaluation of potential protective, low-maintenance coatings for IFE optics. Test coatings of diamondlike carbon (DLC) made by advanced plasma and ion beam methods (ion-beam assisted evaporation and plasma immersion ion plating) were screened for adherence, abrasion resistance, and optical transmission in Phase I. Coatings of undoped DLC proved more durable, adherent, and transparent than did the fluorinated DLC coatings originally proposed, although their optical losses $(\sim 4 \% @ 248 \mathrm{~nm})$ need to be further reduced.

Equipment design concepts are presented for deposition equipment employing a proactive approach to contamination control and the valuable lessons learned in the semiconductor and optoelectronics industries, and using the design methodology for nearultrahigh vacuum systems. This Report presents a roadmap for process optimization and scaleup, and supply of production optical packaging coating equipment and commercial processing services in Phase II and Phase III.

The most promising types of coatings will be applied to actual laser optical components in the Phase II program, and further tested and optimized. Specifications and conceptual designs will be generated for the most successful coating process and associated deposition equipment for scaleup, and implementation in Phase II. If the needed optical component packaging technology can be developed for IFE optics, then it may also useful in important DoD HEL programs including ABL (AirBorne Laser), GBL (Ground Based Laser), SBL (Space Based Laser), ATL (Advanced Tactical Laser), and THEL (Tactical High Energy Laser). 
Film properties related to the ability to resist contamination, including hydrophobicity, roughness, and electrical conductivity, will be measured and correlated to coating process parameters and composition early in Phase II, to aid the selection of the most promising types of coatings for further testing and application to thin-film coatings representative of IFE optical components. Prototype components for typical IFE applications will be treated and delivered to the cognizant DoE laboratories and contractors in Phase II for IFE environmental effects testing.

The Phase II program will provide specifications for a deliverable coating process and turn-key production equipment, engineered with contamination control as a high priority. Phase II activities will include the optimization of the coatings, followed by application to IFE optics to be thoroughly tested by PVD Products, the DoE, and their contractors, and qualified for use in IFE experiments. The coatings' environmental durability and ability to resist contamination will be thoroughly tested and maximized. A dedicated pilot production system will be constructed using ultraclean design rules and in-situ monitoring of contamination and key process parameters, and tested. In Phase III, deliverable processing equipment will be provided to DoE and/or its IFE optics contractors in support of the Integrated Research Experiment (IRE) and eventually the Engineering Test Facility (ETF), while commercial coating markets including precision optics will also be pursued. 


\section{Background}

Inertial Fusion Energy Optics Requirements. Durable reflective coatings (and the associated deposition equipment) are required for the final mirrors directing intense laser beams at targets in developmental fusion energy systems, to address the urgent problem of maximizing the lifetime of the final optical elements on the laser fusion reaction chamber.

In the operation of lasers and reactor chambers for inertial fusion energy (IFE) programs, optical surface damage is a significant problem, particularly for the final optics that admit very high-energy ultraviolet laser beams into the fusion reactor chamber and direct them onto a fusion target. These critical optical components are difficult to shield from energetic particle radiation, and require a durable, stable coating so that they may be used (and cleaned, if necessary) for long periods without damage. This project addressed requirements for a protective coating for IFE final optical components by employing advanced plasma and ion beam deposition methods.

Overcoated reflective coatings of high durability and long lifetime must be developed for application to IFE final optics. Coatings must be optimized, and an ultraclean deposition system designed and constructed to apply protected reflective coatings to prototype and full-scale IFE final optics substrates. Coated optics must then be tested for KrF laser exposure and environmental durability, including exposure to high-energy particles representative of the IFE environment.

General considerations for high-power KrF laser optical components include use of highly polished substrates, and coatings made of materials with high bandgaps and low impurity levels, which must be deposited with a smooth, amorphous or nanocrystalline microstructure. Coating contamination, whether on the surface, within layers, or between layers, must be very low.

The availability of robust packaging methods for coated optical components should have benefits in many areas of applications for lasers, including material processing systems, LIDAR systems, and aerospace systems. Other non-optical commercial applications include data storage, wear and friction reducing coatings, micro-electromechanical devices, microelectronics manufacture, displays, and ophthalmics. 


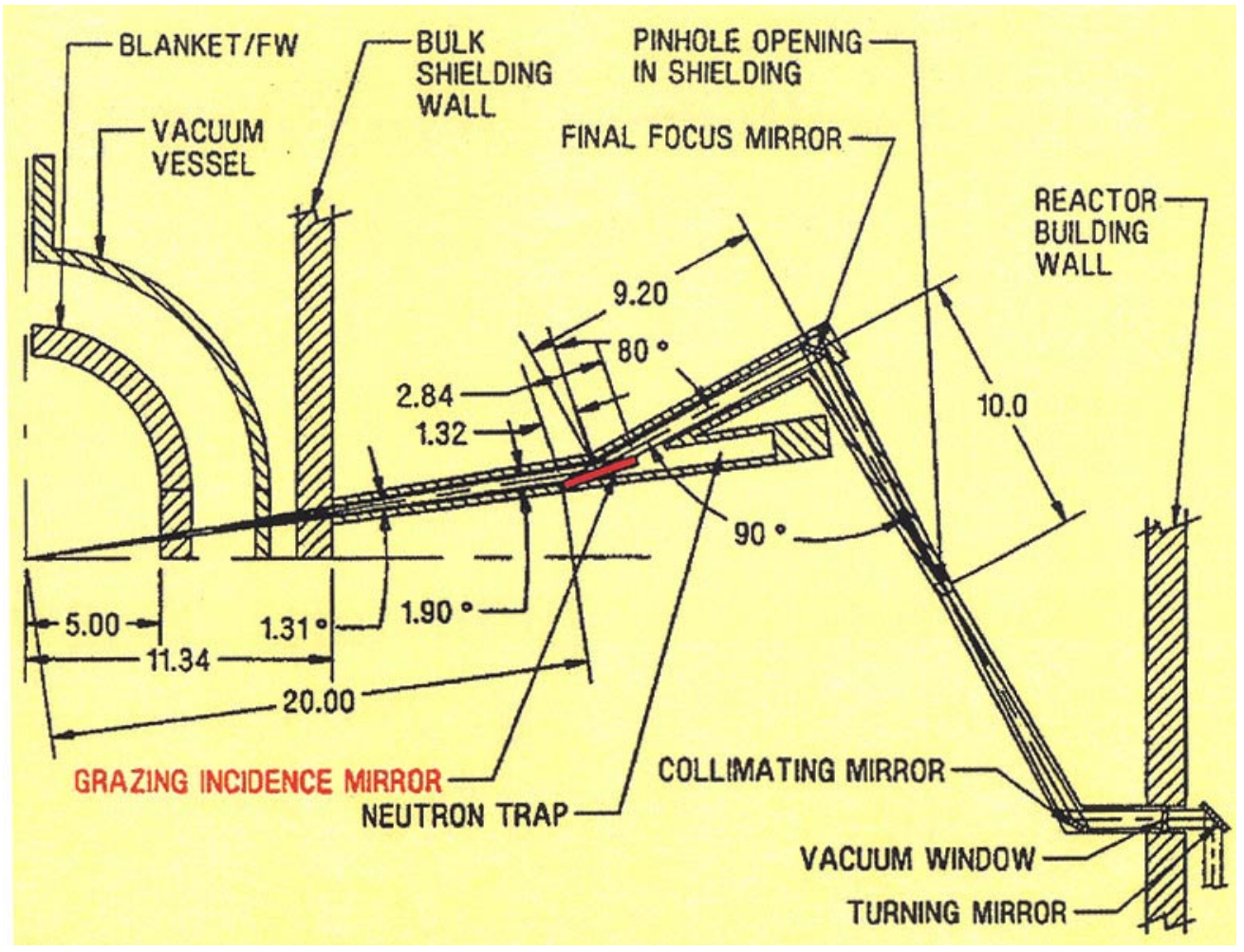

Figure 1. IFE Final Optics Schematic

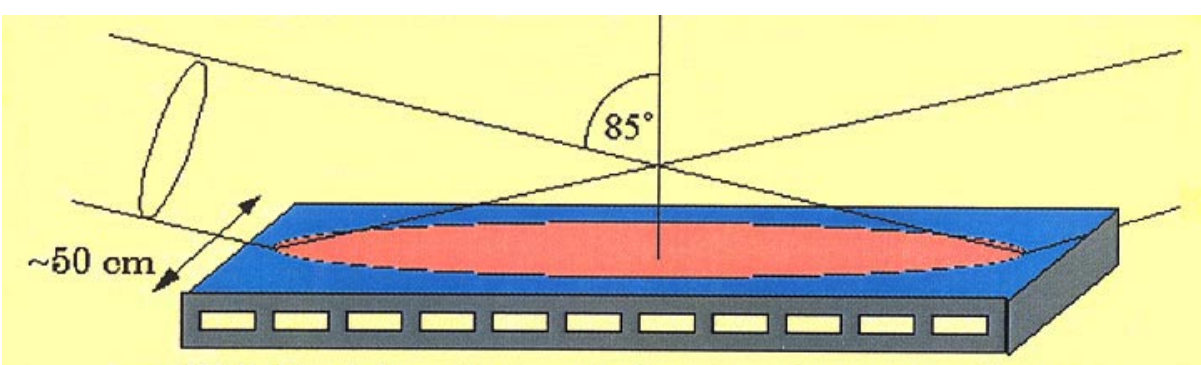

stiff, lightweight, actively cooled, neutron transparent substrate

$\sim 5 \mathrm{~m}$

Figure 2. Concept for Full-Scale IFE Grazing Incidence Metal Mirror (GIMM) 
- The IFE operational wavelength of interest at present is $248 \mathrm{~nm}$ (KrF excimer), and possibly harmonics of solid-state lasers at 266 or $355 \mathrm{~nm}$.

- Full-scale IFE final optics components may be up to 1 meter or more in length. Designed to minimize weight while maintaining stiffness, they may consist of relatively thin polished crystals of CVD Silicon Carbide, coated with Aluminum, and used at grazing incidence angles of a few degrees.

- Neutrons

- UV photons

- X-ray photons

- Gamma rays

- Energetic ions

- Target Debris

- Contamination

Figure 3. Hazards of the IFE Environment

- Material removal (ion sputtering, $X$-ray ablation)

- Ion implantation

- Densification

- Swelling

- Formation of color centers and other defects

- Changes in optical properties

- Destruction of interlayer interfaces

- Surface roughening

- Decomposition of compounds

- Transmutation

- Desorption and redistribution of contamination

Figure 4. Negative Effects of IFE Environment on Final Optics 
Desirable properties of IFE final optics coatings. Coatings must have very low absorption and be thin, uniform, and have no lower laser damage threshold than either bare substrates or coated laser optics. From a practical standpoint, the coatings should be reliable, durable, and capable of a long service lifetime with a minimum of maintenance. Stability, adherence, and compatibility with existing and future laser optics are essential. Thus, the qualities of an IFE final optics coating must include:

- Excellent adherence

- High UV laser damage threshold

- Very high purity (absence of absorbing impurities)

- Very low defect density (pinholes, voids, and inclusions)

- Low optical scatter (smooth microstructure)

- Low optical absorption

- Low stress

- Low maintenance requirements

- Cleanable without damage (physical and chemical durability)

- High stability and long service lifetime

- Resistance to fusion environmental effects (energetic photons, neutrons, ions, etc.)

- Manufacturable by robust, ultra-clean process

- Scalable to industrial production of full-scale IFE laser optics

Figure 5. Properties of an Ideal IFE Final Optics Coating

It would also be useful if the coated surface were hydrophobic. This would discourage the condensation of water vapor on the surface, which tends to bring other contaminants along with it that remain on the surface even after the water evaporates. 
However, the IFE optics packaging coating must not reduce optical component performance. Thus, a successful protective overcoating must not degrade the underlying optical surface, by contributing either:

- Roughness, which causes undesirable optical scattering

- Contamination, which causes many problems including increased optical absorption and decreased coating adherence

- Stress, which causes optical distortion and decreases adherence

- Non-uniformity, which degrades mirror figure, beam quality, and focusing

- Defects, which drastically lower local laser damage thresholds and can lead to catastrophic coating failure

\section{Figure 6. Undesirable Properties of IFE Optical and Protective Coatings}

It should also not be adversely affected by the many harsh environmental factors inherent in the context of IFE reactors, including energetic radiation, particles, and target debris.

A successful IFE optical coating should be robust, stable, and chemically inert. It must protect critical optical surfaces from degradation due to both typical contaminants and the cleaning materials and methods used to remove them. The coating must not require frequent cleaning, which only increases the chance the component will be damaged.

Protective overcoatings for aluminized IFE final optics reflecting surfaces must themselves have very low optical absorption (ideally $<0.1 \%$ ) and be thin, uniform, and have no lower laser damage threshold than either bare or coated substrates. When applied to an already-coated IFE optical component, an acceptable packaging coating must not contribute significant increases in surface roughness or contamination, nor distort the substrate. From a practical standpoint, the coatings should be reliable, durable, and capable of a long service lifetime with a minimum of maintenance. Stability, adherence, and compatibility with existing and future IFE optics are essential. 
Based on the best available information at the present time, the preliminary specifications of the IFE final optics reflective coating are listed below:

Coating: Overcoated Aluminum, possibly with an underlayer* as well

Materials:

Overcoating - probably Aluminum Oxide or Silicon Dioxide

Thickness - effective half-wave at $248.4 \mathrm{~nm}$, for grazing incidence at $85^{\circ}$ from normal

Reflective layer - Aluminum; thickness TBD, may range from 500A to 1-2 microns

*Undercoating - TBD (if needed) might possibly be Titanium Silicide, Palladium, Titanium, or Nickel; Thickness - TBD

Purpose - a buffer layer to provide a less abrupt transition between the very different properties of $\mathrm{Al} \& \mathrm{SiC}$ (stiffness, thermal expansion coefficient)

Substrates: probably Silicon Carbide, approx. 4" dimensions ( 6" substrate platter) (Possibly made from monolithic Aluminum)

Processes:

Ion Beam Cleaning

- bare substrate

- remove oxide from Al layer before overcoating deposition

- possibility of removing coatings from substrate

- possibility of smoothing substrates and/or coated optics

DC Magnetron Sputtering

- Al reflective layer

- Undercoating layer (if it is a metal)

RF Magnetron Sputtering

- Overcoating layer

- Undercoating layer (if it is a dielectric)

Ion Beam Assisted Deposition

- Undercoating layer (if required)

- Al reflective layer

- Overcoating layer 
Effects of Contamination on High-Energy IFE Laser Optics. A significant limitation on the effectiveness, lifetime, and reliability of high-energy laser optics in realworld situations is the accumulation of surface contamination, eventually requiring some type of difficult and risky maintenance operation. At low levels contamination reduces laser performance (and reliability) due to increased scatter and absorption losses particularly important for reflective optics, where light must pass twice through any surface contaminant film. At higher levels, contamination leads inevitably to catastrophic laser damage of critical optical surfaces. Localized contamination may become a site of catastrophic damage, while the buildup of diffuse contaminant layers leads to a slow loss of efficiency. In-situ maintenance may thus be required so that an IFE laser system with contaminated optics can be used without substantially damaging itself. NASA planetary and space astronomy missions have similar problems (1), as do space optical systems for critical DoD applications including surveillance and ballistic missile defense. Thus, current high-energy laser optical components have extreme vulnerability to contamination, and in particular will frequently suffer considerable damage from even careful attempts to remove contamination (if it is allowed to occur).

Prevention of IFE optics contamination before it occurs must be the paradigm for truly practical operation of IFE systems.

- Coating surface degradation

- Increased optical losses (absorption and scattering)

- Susceptibility to laser damage

- Reduced lifetime and reliability

- Increased maintenance

- Cleaning-related damage

Figure 7. Contamination Effects on Laser Optics

A number of improvements in IFE optics are needed, to increase their lifetime and performance, and to reduce their need for maintenance, as well as their susceptibility to damage from high fluences of $\mathrm{UV}$ photons and other energetic particles (neutrons and ions). Their surfaces must be modified if possible to discourage the condensation of water vapor and the sticking of other contaminants, including particles, but also to allow the coated optics to be cleaned without damage when absolutely necessary. What is needed is a deterministic, practical engineering approach to packaging coated IFE optics, analogous to successful IC manufacturing methods. 
Contamination Control. An integral and ongoing part of this program is the high priority of controlling contamination in the eventual handling and coating of larger IFE optical components. Because of the difficulty of complete removal of contamination from delicate polished surfaces without appreciable damage, the prevention of contamination will the operative paradigm for equipment design and construction material selection, process design, and procedures for inspection, cleaning, handling, and storage of uncoated and coated substrates. A contamination control specification will be developed and followed for both the prototype and larger pilot production coating systems, based in part on the SEMATECH "Guide for Contamination Control in Design, Assembly, and Delivery of Semiconductor Manufacturing Equipment” (SEMASPEC \#92051107A-STD, summarized below in this Report, and other relevant documents.

Critical to the success of the proposed concept for IFE optics coating is the dominant role of contamination control. A pro-active approach to the problem must have the goal of preventing the occurrence of contamination, so that its removal will not even be necessary. The application of even the best possible optics packaging coating is only a part of the overall contamination control approach required, which must succeed in all of its elements, or it fails to be truly effective:

- $\quad$ Equipment design and construction material selection

- $\quad$ Process design

- Handling and storage procedures

- Cleaning and inspection procedures

- $\quad$ Facility cleanliness

- Clean room procedures and training

Figure 8. Elements of Contamination Control Program

Even employing the best possible optics packaging coating will not make up for deficiencies in these areas. Consequently, a detailed consideration of contamination control must guide the development of conceptual designs and specifications for ultraclean coating processes and equipment for IFE final optics coatings. 
Fluorinated Diamondlike Carbon Coatings. The original rationale for this program was that most, if not all of the abovementioned coating requirements could be satisfied, to the extent that it might be possible to combine the best features of diamond and fluorocarbons (e. g. Teflon) in the same coating, which in the most favorable outcome could then be described as a "hard plastic". Both materials are chemically inert, and have good surface-protection and passivation capabilities, high optical bandgaps and a wide range of transparency (2) and resistance to space environmental effects (which are quite relevant to the hazards of the IFE environment).

Diamond's high inter-atomic bond energy provides its extreme hardness, as well as high abrasion and radiation resistance, and general physical and chemical durability. However, the hardest diamond films are usually highly stressed.

Teflon's high contact angle for water would minimize the condensation of water-borne contamination on IFE optics, while its very low refractive index (about 1.3) should allow its use as an outer layer on antireflection-coated windows and lenses without increasing surface reflection losses. However, Teflon is a very soft material.

If a fluorinated, diamond-like carbon coating can be deposited in amorphous form, it may have very low optical scatter losses and low stress, as well. The desirable properties expected of F-DLC are summarized below:

- Very Low Surface Energy, High Water Contact Angle

- Hydrophobic Surface

- Chemically Inert

- Low Friction

- Optically Transparent, High Bandgap, Low Refractive Index

- Near-Room Temperature Deposition Process

- Compatible with Many Substrate Types

- Low Stress

- Low Roughness Surface

- Protective Barrier Against Water Penetration

- Resistant to UV Radiation

- Usable at Cryogenic Temperatures

Figure 9. Properties of Fluorinated Diamondlike Carbon (F-DLC) Coatings 


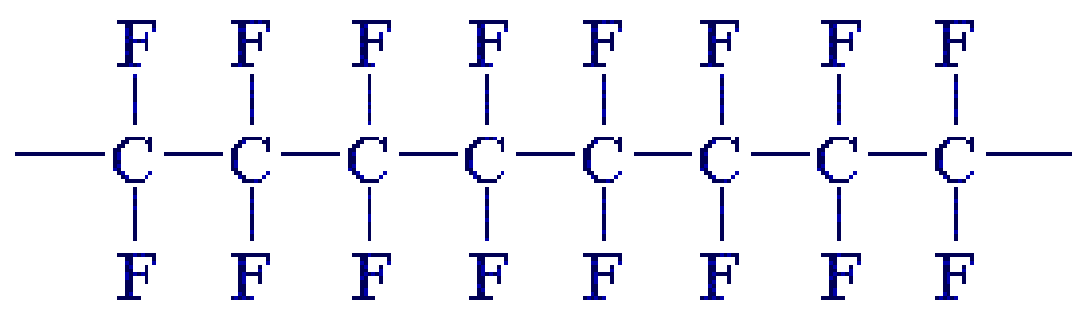

Figure 10. Fluorocarbon Polymer Structure

Because of fluorine's high electronegativity, very strong carbon-fluorine bonds hold the $F$ atoms tightly. The $F$ atoms effectively shield the carbon backbone of fluorocarbon polymer molecules such as Teflon. Thus the molecule has a fluorine "surface", which has a very low surface energy, making interactions with other molecules energetically unfavorable, and so the surface is "non-stick" and chemically inert. In fact, fluorocarbons are the most hydrophobic of common organic compounds (3), so the condensation of water on such surfaces is considerably inhibited.

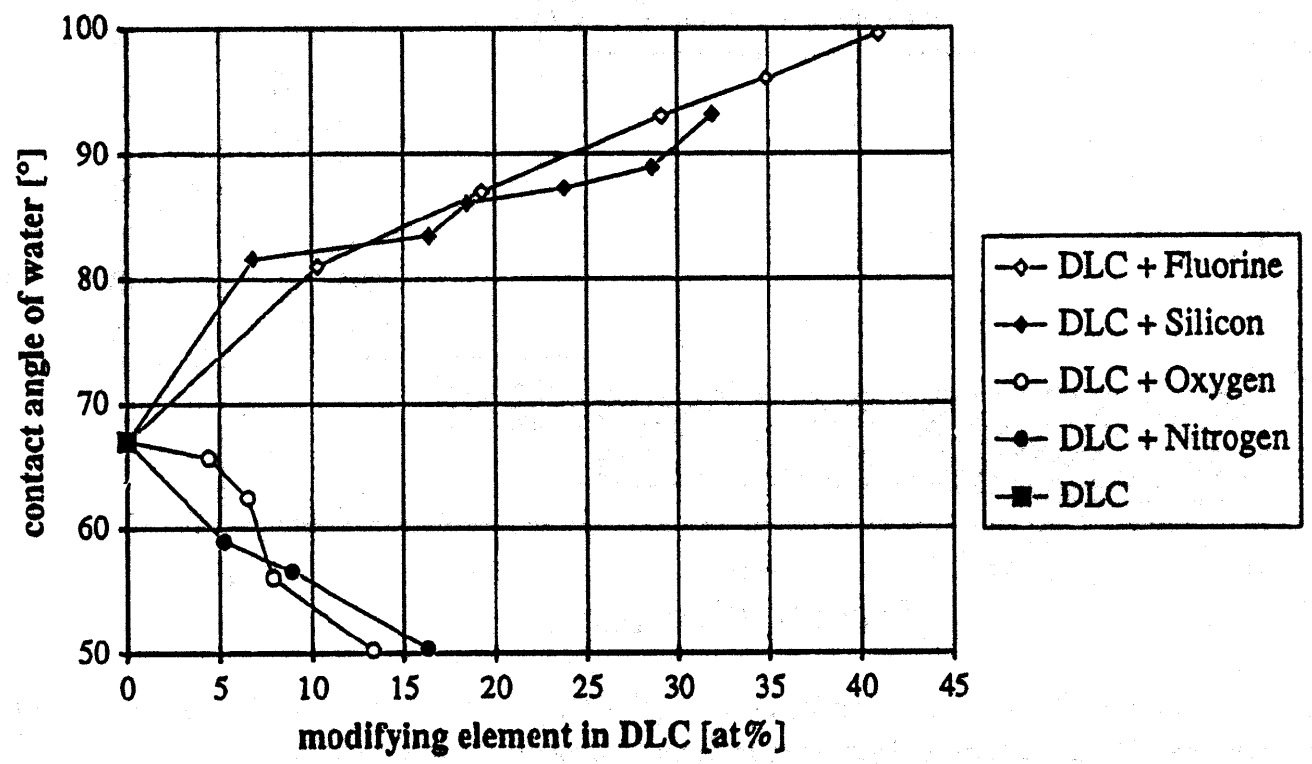

Figure 11. Change in the Contact Angle of Water by Adding Different Elements to DLC Coatings (Reference 4) 
It may be possible to increase the contamination-repellent ability of the proposed IFE optics packaging coatings by modifying the important surface properties of F-DLC coatings, including water contact angle (4) and electrical conductivity (5) by adjusting the fluorine content. With increasing fluorine content, there are increases in water contact angle, bandgap, and optical transmission; quantities that decrease with increasing $F$ content include optical loss, stress, density, and hardness. At very high $F$ content, a point of diminishing returns is encountered, so it is anticipated that the optimum $F$ content will be determined by a tradeoff among the various film properties of importance.

DLC, like Teflon, is a very low surface energy material, whose high contact angle for water inhibits the wetting of its surface by condensation of water vapor. Water condensation on DLC can be reduced even further by addition of "dopants" such as Fluorine and/or Silicon (4), while Oxygen and Nitrogen doping have negative effects on the water contact angle (see Figure 11).

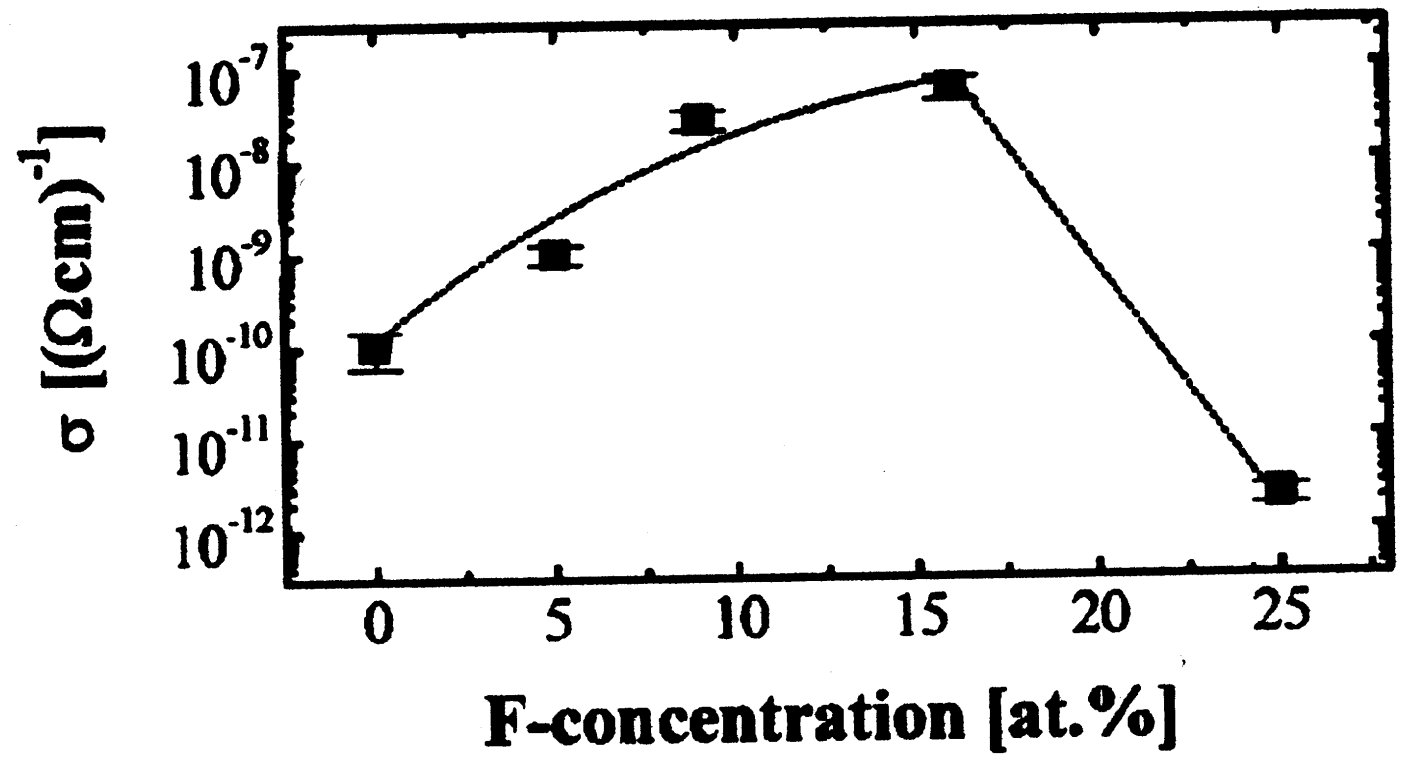

Figure 12. Electrical Conductivity of Amorphous C:F Films as a Function of Fluorine Concentration (Reference 5)

Possibility of Repulsion of Particles. Addition of Fluorine (or other dopants) to Diamond coatings may increase their electrical conductivity by two to three orders of magnitude, in concentrations of 10-15 atomic\% $F$ (see Figure 12). This suggests that $F$ DLC-overcoated optics would not tend to acquire and hold a surface charge, compared to conventional (low-electrical conductivity) dielectric-coated laser optical component surfaces. Although this type of coating might not actually repel particles, their electrostatic attraction to the surface and subsequent accumulation may be considerably reduced.

Moreover, the use of such an outer coating layer with appreciable electrical conductivity would allow the possibility of imposing a desired electrical potential (i. e. a bias voltage) on 
the outer coating surface. In principle, the bias voltage could be adjusted to repel particles with a charge of the same polarity. (Generally, particles are positively charged, as they lose electrons more easily than they gain them.) This possibility will be investigated in the Phase II program, based on electrical conductivity measurements of test coatings.

Optical Coating Durability Specifications. Existing US military specifications for the mechanical and environmental durability of coated optical components include tape pull tests for adherence, eraser rub tests for abrasion resistance, humidity and salt fog exposure, and temperature cycling. Potentially relevant specifications include sections of MIL-C-675, MIL-C-14806, MIL-C-43454, MIL-C-48497, MIL-E-12397, MIL-F-48616, MIL-M-13508, MIL-O-13830, MIL-P-16898, MIL-STD-810, and A-A-59199. Many of these specifications were written before the advent of high-energy lasers, and may have to be applied selectively, or updated as required. Other relevant environmental factors include thermal and mechanical shock, as well as laser damage resistance in actual use. An ongoing activity of this program is to identify the existence and relevance of pertinent civilian and military specifications, as well as those promulgated by organizations including ASTM, NIST, EIA, NASA, the LIGO gravity-wave detection project, and the high-energy laser programs at the national laboratories and the University of Rochester. This is an essential foundation for producing a good specification for a robust, low-maintenance coating for IFE optics.

Typical MIL-Spec tests presently required to document the environmental resistance and durability of commercially available optical coatings include:

- Temperature (per MIL-M-13508 C, paragraph 4.4.4)

5 hours exposure at both $-62^{\circ} \mathrm{C}$ and $+71{ }^{\circ} \mathrm{C}$

- Hardness (per MIL-M-13508 C, paragraph 4.4.5)

50 strokes with a cheesecloth

- Adherence (per MIL-M-13508 C, paragraph 4.4.6)

"Scotch" tape pull test

- Humidity (per MIL-M-13508 C, paragraph 4.4.7)

24 hrs exposure at $49^{\circ} \mathrm{C}$ and $95 \%$ humidity

Figure 13. Military Specifications for Environmental Durability of Coated Optics 
Deposition Processes. A desirable coating process for application of IFE final optics packaging coatings should allow relatively low-temperature $\left(<150^{\circ} \mathrm{C}\right)$ deposition of very smooth, amorphous, uniform coatings by a clean, economical, scalable process, in cleanroom compatible equipment with a minimum potential for either particulate or volatile contamination. The coatings produced should have low stress, and be essentially free from defects and impurities, and compatible with existing and planned IFE optics requirements.

A dense, pinhole-free coating is required, which implies that arriving atoms of the coating material have good lateral mobility across the substrate. Such diffusion during the coating process minimizes the formation of voids and other structural defects, and can be achieved by substrate heating (which is often undesirable) or the involvement in the process of energetic atoms or ions (which may be of the coating material, or a different inert or reactive element).

A number of methods have previously been used to deposit DLC coatings, including chemical vapor deposition and plasma and ion beam processes (5-9). Significant work in development of ion-beam deposited diamondlike carbon coatings has been done by several laboratories, including the Army Research Laboratory under the direction of James K. Hirvonen and Kostas Fountzoulas, the Southwest Research Institute by Geoffrey Dearnaley and James Arps, and NASA Glenn Research Center, Electrophysics Branch, by Bruce Banks, Sharon (Rutledge) Miller, Michael Mirtich, et al.

Diamondlike carbon coatings have been studied for military and other protective applications with encouraging results by several investigators (14-37); this work has laid the foundation for the improvements proposed in this program. Development of silicated (38-43) and fluorinated (5-9) DLC coatings has been reported on by several laboratories. Other important work in the area of fluorinated carbon coatings has been done in the US at LANL by the Nastasi group (7), and by IBM, Stanford, Texas Tech, and others, while overseas contributions $(4,5,8,9)$ have been made at the University of Gottingen and the Fraunhofer Institute in Germany, the J. J. Thomson Laboratory in the UK, CSIRO in Australia, IDF in Brazil, and several laboratories in Japan. (The assistance of Dr. James K. Hirvonen of the Army Research Laboratory in compiling references for this project is gratefully acknowledged.)

The coating methods chosen for investigation in the Phase I program were advanced plasma and ion beam methods that involved a low substrate temperature, and were judged to have a reasonable possibility of being scaled to the treatment of meter-size and larger optical components. They include Ion-Beam Assisted Evaporation and Plasma Immersion Ion Processing, using fluorocarbon and/or hydrocarbon coating precursors and Argon as the working gas. A brief description of the deposition processes follows: 
Ion-Beam Assisted Deposition (IBAD). This process involves the interaction of two beams at the surface of the substrate, to make a dense, adherent coating: 1) A beam of neutral, very low-energy $(<<1 \mathrm{eV})$ atoms and molecules from an evaporation source, and 2) A beam of higher-energy ions (typically Argon) and electrons from an ion source.

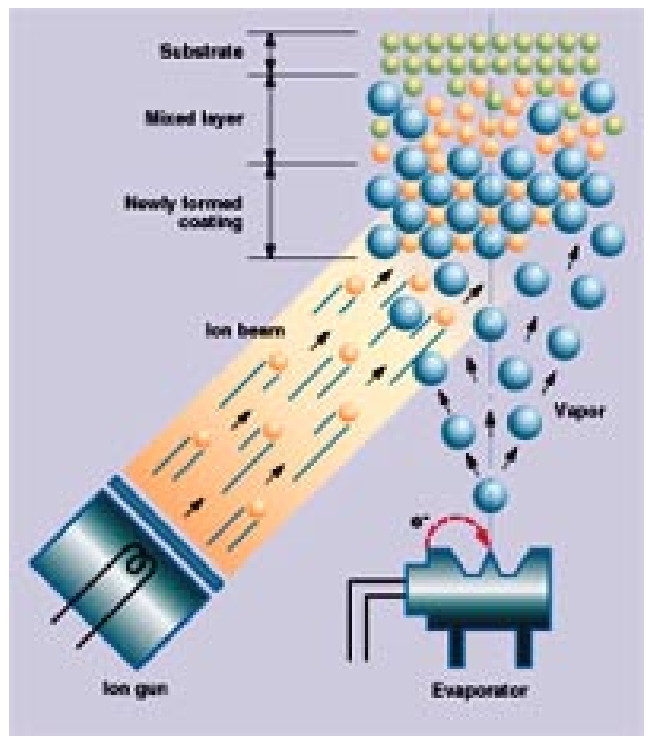

Figure 14. Deposition of Diamondlike Carbon (DLC) by Ion Beam Assisted Evaporation of Liquid Hydrocarbon Precursors (SwRI illustration).

The suprathermal kinetic energy of the ions (from a few hundred to a few thousand electron Volts) provides several beneficial effects on film quality:

- Surface cleaning and creation of nucleation sites (which improves film adherence)

- Increased lateral mobility of arriving film atoms on the substrate surface (leads to denser films)

- Densification of the growing film by momentum transfer from the ions to the film atoms

- Removal of loosely bonded film atoms

Figure 15. Beneficial Effects of Ion Bombardment on Film Quality

Thus, a much better coating can potentially be made with concurrent ion bombardment than by evaporation alone $(10,11)$. These benefits occur to some degree in all ion beam and plasma deposition processes, including both Ion Beam Assisted Deposition (IBAD) and Plasma Immersion Ion Processing (PIIP). However, the use of ion bombardment to produce low-loss optical coatings for deep UV wavelengths should be done carefully, to avoid increasing the scattering and/or absorption of incident UV photons. 
Plasma Ion Immersion Processing (PIIP). In the PIIP process, a plasma consisting of precursors of the desired coating material is created in a vacuum chamber, in proximity to a substrate whose holder is:

- electrically isolated from the chamber itself

- connected to a power supply which allows a desired electrical potential (bias voltage) to be impressed on the substrates

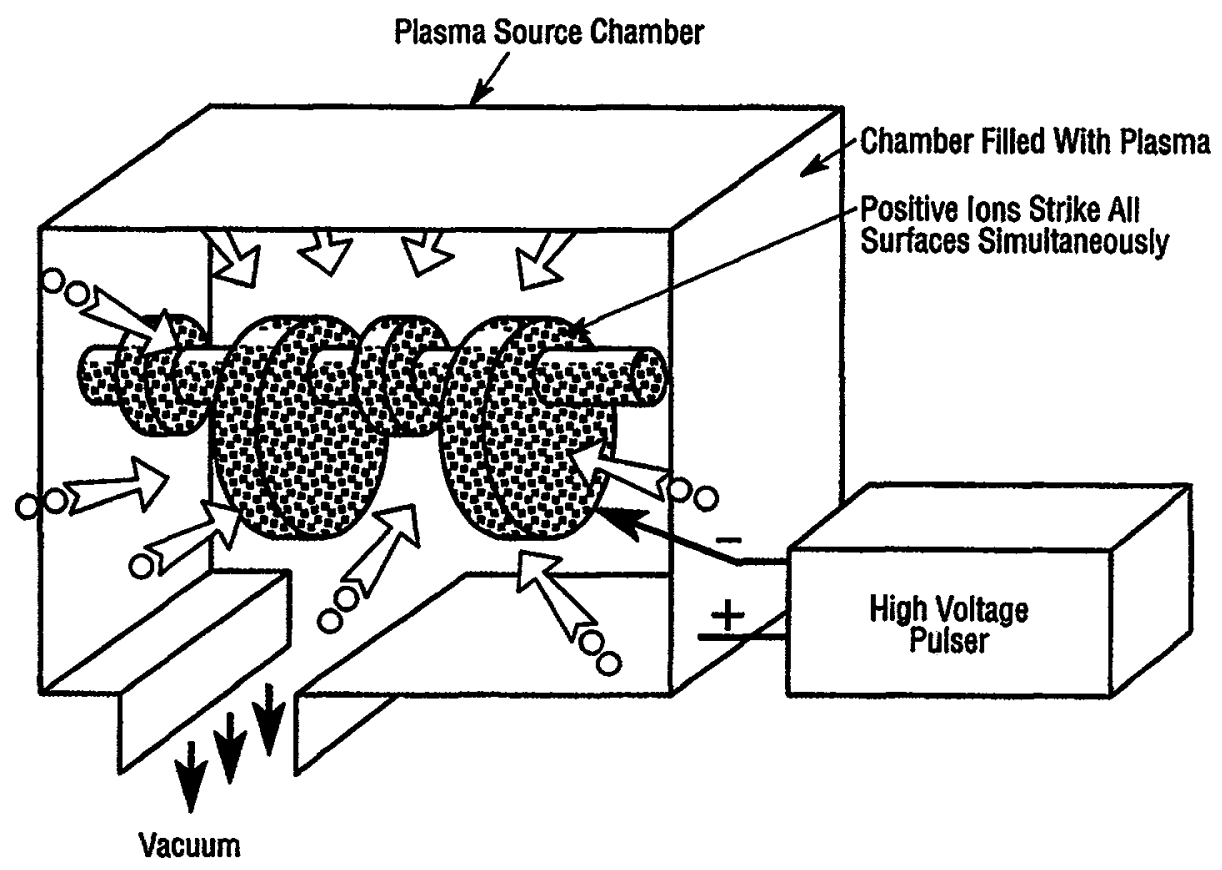

Figure 16. Illustration of Plasma Immersion Ion Processing (PIIP) process (Reference 12)

"PIIP utilizes a time-varying (pulsed) bias on a target to extract ions out of plasma for ion implantation and coating deposition. Plasmas can be made by capacitive or inductive radio frequency sources or by initiating a glow discharge during each pulse of high voltage. Plasmas of hydrocarbon gases have been used to deposit adherent diamond-like carbon (DLC) coatings on a variety of ferrous and non-ferrous materials. To improve the adhesion of DLC, PIIP uses ion implantation to create a graded interface between the substrate and the DLC coating." - quotation from Reference 13 (See also Reference 14.)

In the Phase I program, PIIP coatings of fluorinated, silicated, and undoped DLC were produced at the Southwest Research Institute (SwRI) by Dr. James Arps and Rong-Hua Wei. 
Background on PIIP process (reference: SwRI). Plasma immersion ion processing (PIIP) is a relatively new vacuum technology for the application of hard, wear-resistant coatings. Like conventional physical vapor deposition (PVD) methods, PIIP is used to deposit various coatings, but the non-line-of-sight PIIP approach allows simultaneous treatment of large components and complex shapes without requiring component manipulation. The recent availability of inexpensive, high-power pulsed modulator equipment, and the inherent scalability of PIIP, lend an economy to this surface modification process which is difficult to match using other methods. PIIP technology was originally developed at the University of Wisconsin (by John Conrad et al) and principally refined at Los Alamos National Laboratory (LANL) by Michael Nastasi, Kevin Walter, and co-workers, and is being developed for Government and commercial applications by SwRI.

The steps in the PIIP process are as follows:

- Components are inserted into vacuum chamber

- Chamber is evacuated to base pressure below $10^{-5}$ Torr

- Plasma (mixture of ions and electrons) is generated from gas introduced into chamber

- All exposed surfaces are completely surrounded by plasma

- High-voltage pulse modulator generates negative bias voltage pulses applied to components

- Positive ions are extracted out of plasma toward components

- Ions impinge on all exposed surfaces of components simultaneously

- Voltage pulses $>20 \mathrm{kV}$ are used to produce ion implantation

- Voltage pulses $<10 \mathrm{kV}$ are used to deposit coatings

- Ion implantation or interlayer deposition can be used to improve coating adherence

Figure 17. Steps in PIIP Deposition Process

The versatility of the plasma immersion approach to surface modification allows the use of any gas with the same equipment. Thus, a single vacuum chamber can use argon gas for sputter cleaning, nitrogen gas for ion implantation, and/or hydrocarbon gases or organometallic compounds to deposit coatings.

PIIP techniques for the deposition of diamond-like carbon (DLC) from acetylene gas and chromium oxycarbide from chromium carbonyl have been demonstrated. Researchers 
at LANL have demonstrated the scalability of PIIP by simultaneously coating a $16 \mathrm{~m}^{2}$ substrate area with DLC. Analysis of a large-scale PIIP-based DLC deposition process indicates that the treatment cost would be comparable to electrodeposited hard chrome. Thus, PIIP offers an affordable alternative to more expensive and smaller-scale processes for the deposition of coatings for tribological (wear and friction) applications.

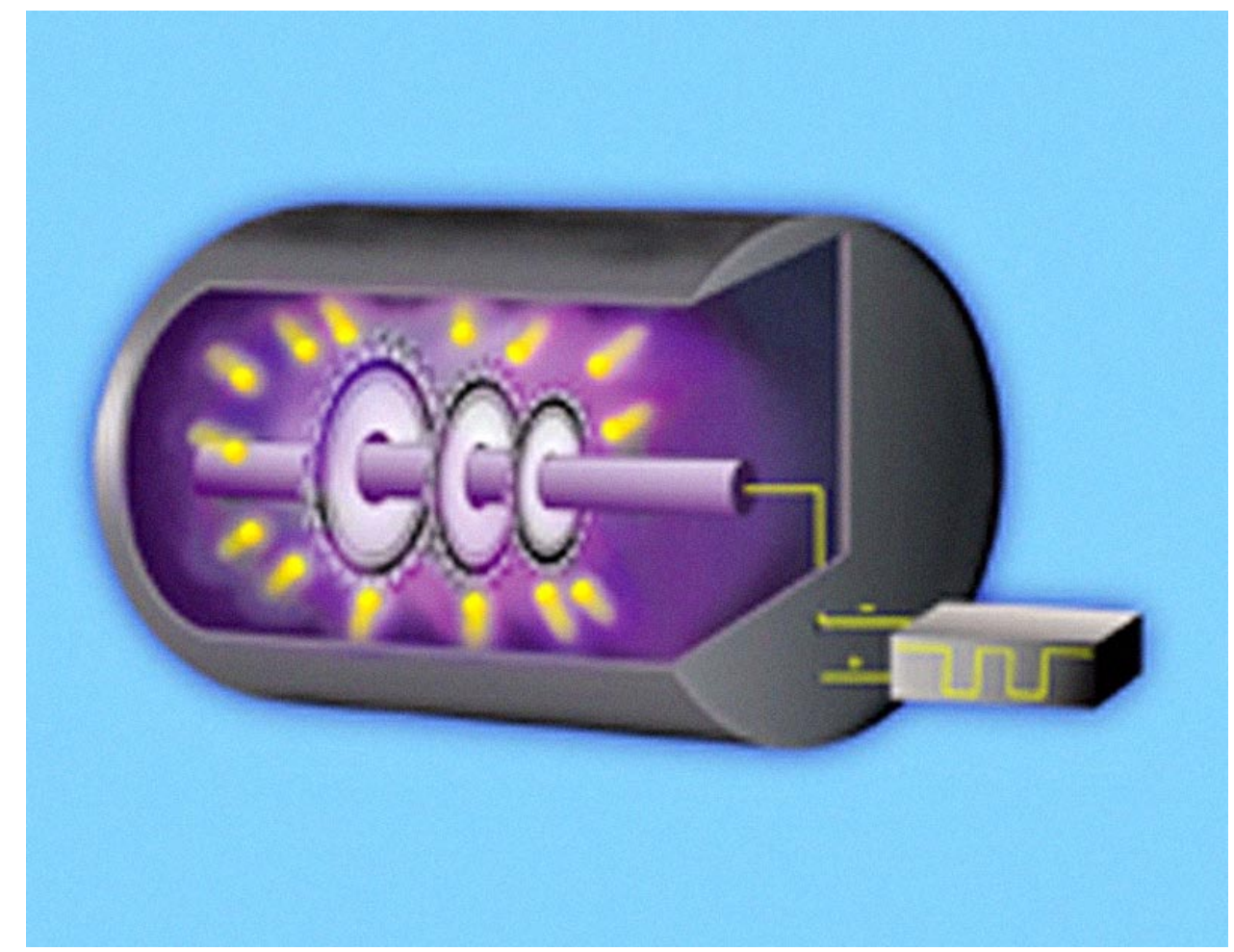

Figure 18. Depiction of Plasma Immersion Ion Processing (SwRI)

The large-area, non-line-of-sight PIIP process has a number of potential advantages over conventional vacuum coating processes, including higher throughput, lower cost, reduced component heating, and the ability to treat all external surfaces of parts simultaneously, without manipulation. 


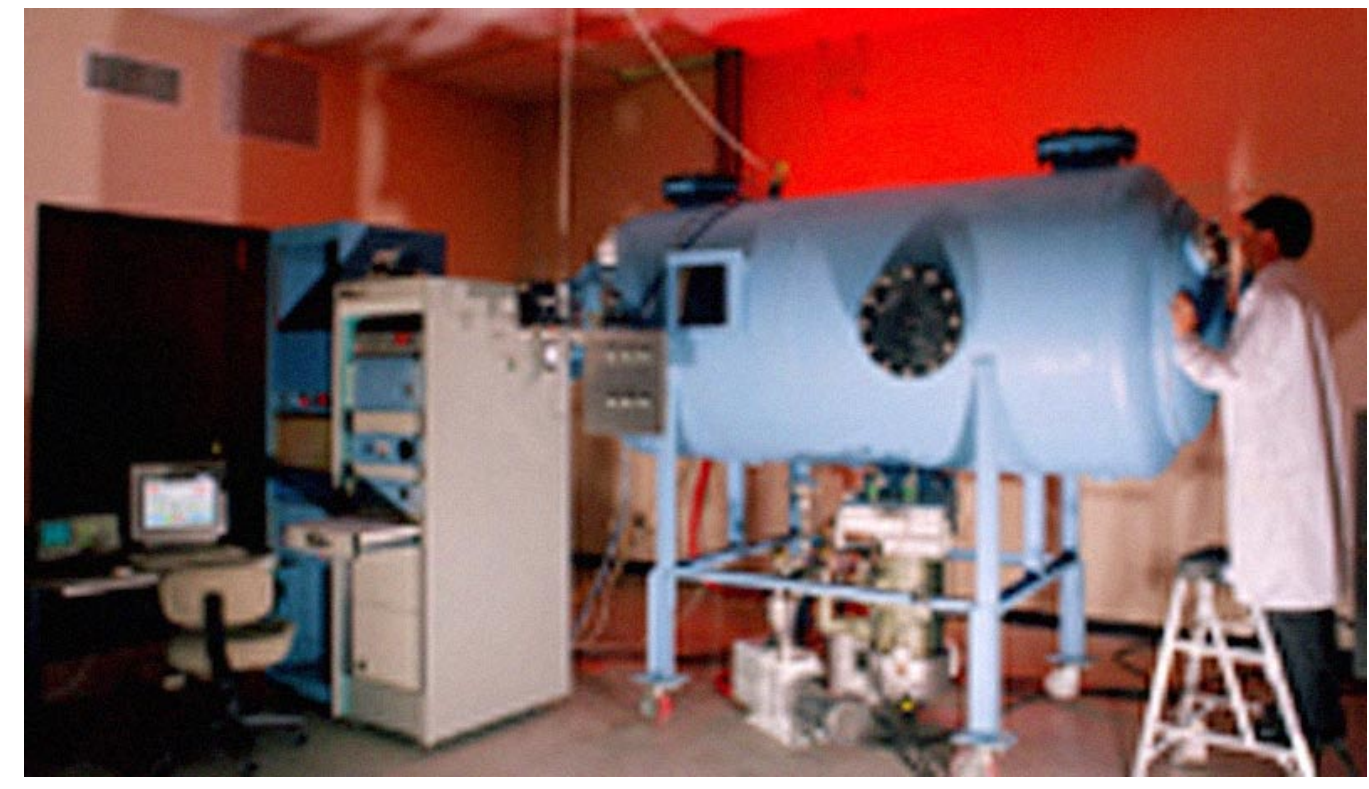

Figure 19. Exterior view of PIIP System at Southwest Research Institute.

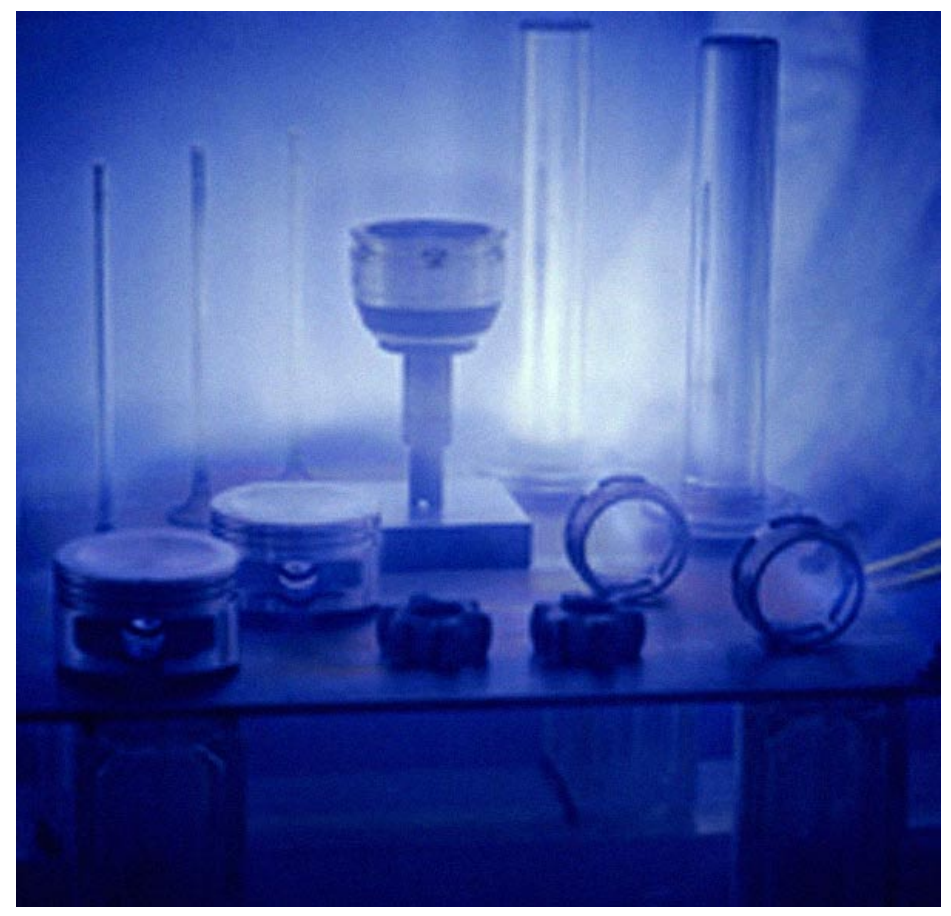

Figure 20. Interior View of SwRI PIIP System during Deposition. 
Coating Process Parameters. The parameter space for coating optimization includes the selection of coating process, which may include one or more of:

- Pre-deposition ion beam cleaning and smoothing of substrates

- Conventional evaporation

- Glow discharge sputtering

- Ion beam-assisted evaporation

- Ion beam-assisted sputtering

- Hybrid evaporation/sputtering/ion beam processes

- Plasma immersion ion processing

- Post-deposition ion beam smoothing

The use of ion bombardment simultaneous with coating deposition offers the ability to optimize the coating microstructure for surface smoothness and a fine-grained, microcrystalline film without significant grain boundaries.

Key deposition process parameters include:

- Coating thickness

- Deposition rate

- Ion beam current density

- Ion energy and species

- Background pressure

- Substrate temperature

- Process pressure 
PVD Products, Inc. PVD Products has designed and constructed several types of advanced coating deposition systems, utilizing methods including sputtering, ion beam processes, pulsed laser deposition, and evaporation, as shown in Figures 21 and 22. While PVD Products was a division of Epion Corporation under the direction of Dr. James Greer (President and Chief Technical Officer of PVD Products), an advanced large-area PLD deposition system for superconducting materials was supplied to the Air Force Research Laboratory at Wright-Patterson AFB.

Prior to starting PVD Products, Dr. Greer conducted research at Raytheon Corporation, and directed advanced coating programs including pulsed laser deposition, and magnetron and ion beam sputtering; he has also worked on a variety of thin film deposition processes for diverse applications, including IR detectors, flat panel displays, and wear resistant coatings, as well as for ferroelectric and magnetic applications, and was also responsible for both prototype production and research relating to the fabrication of unique surface acoustic wave devices for high-performance military applications.

PVD Products facilities in its 6,700 sq. ft. headquarters in Wilmington, MA include 900 sq. ft. of Class 10,000 clean room laboratory space that houses a PVD Products PLD-5000 laser deposition system with an Lambda Physics LPX-220i excimer laser, and characterization equipment including an AMRAY Model 3600 LEAP field emission SEM, a Varian Cary 219 UV-visible-near IR spectrophotometer, a Rudolph Research AutoEL ellipsometer, and a Dektak 8000 surface profilometer. PVD Products also has a $3 \mathrm{~cm}$ IonTech Kaufman-type ion source for ion-assisted deposition processes. Two laminar airflow wet chemical processing stations are also housed within the lab. PVD Products has a mobile modular 275 square foot Class 100 clean room, as well as 2,000 sq. ft. of manufacturing space. Partner facilities for characterization include a custom-built fourpoint probe system, a Digital Instruments atomic force microscope, a Perkin-Elmer model 1600 FTIR spectrometer system, and a 1200X Nomarski photographic microscope. 

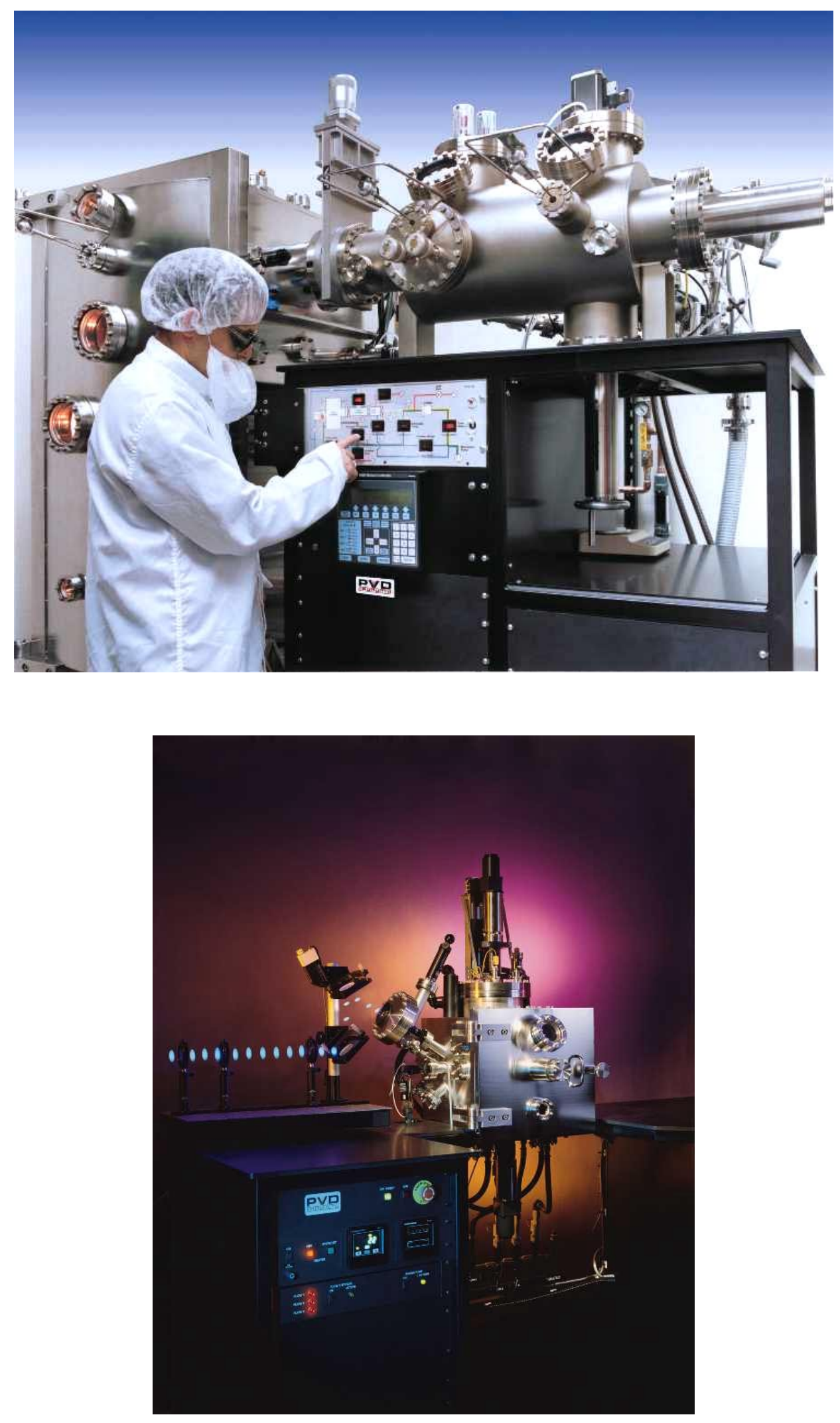

Figure 21. PVD Products Deposition Systems 
Figure 22 depicts a reel-to-reel production deposition system for coating continuous 100 meter tapes by pulsed laser deposition, with separate entry and exit load-lock chambers, three 6-inch material targets, a black body substrate heater, and fully automatic computer process control. With the use of a conveyor-type pallet drive, IFE optics substrates could be processed continuously in production in a multiple-load lock system with ion and material sources positioned sequentially along the direction of motion.

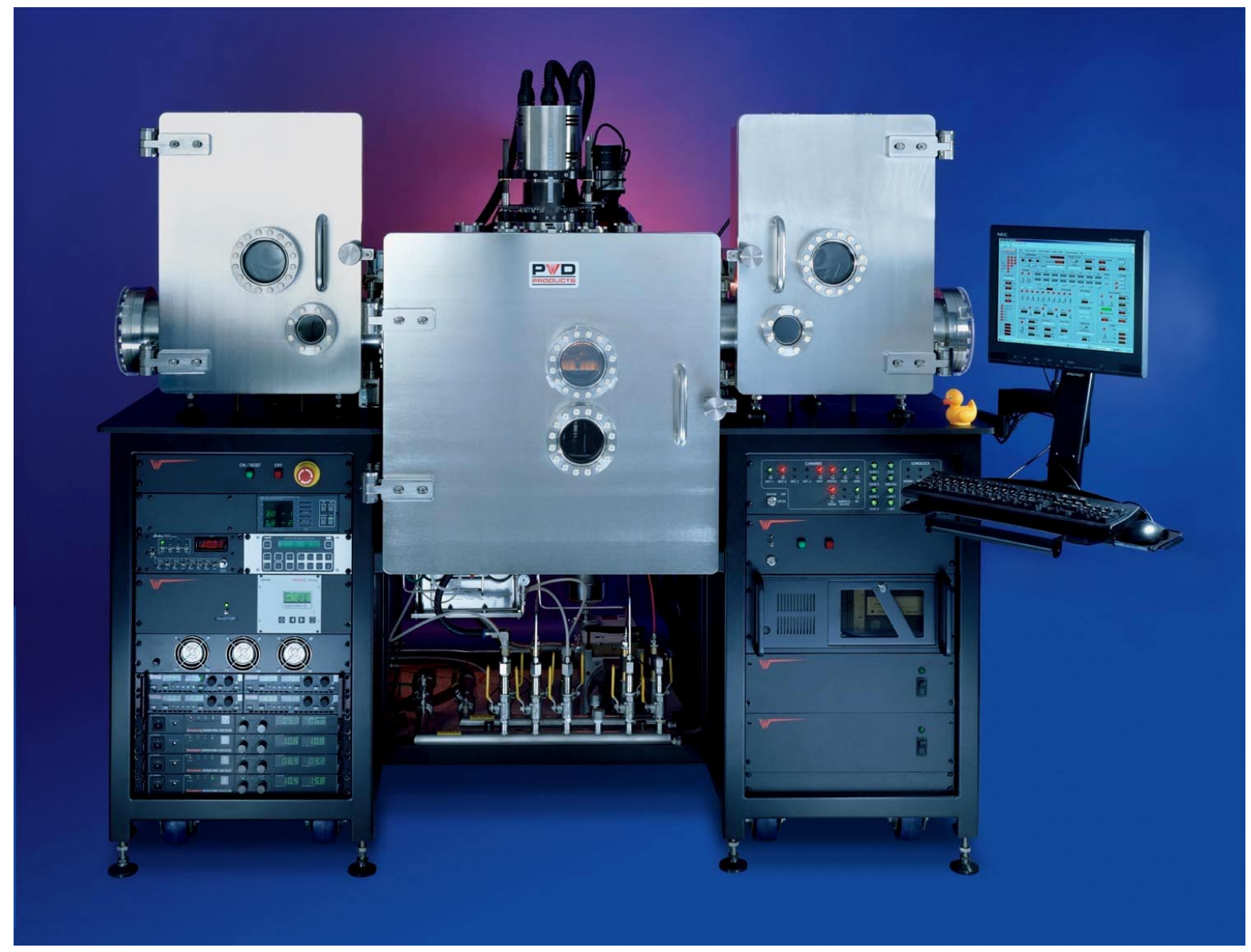

Figure 22. PVD Products Reel-to-Reel Tape Coating System 


\section{Experimental Results}

Phase I Experimental IBAD System. Design criteria for the system for depositing DLC test coatings at PVD Products included a small evacuated volume, smooth electropolished interior surfaces, and process flexibility. Figure 23 depicts the process chamber with internal substrate platform and external rotary drive, the gate valve and top of the pumping stack; the ion beam and deposition sources are not show in this view, but are mounted on flanges at the ends of three tubes which intersect the axis of the process chamber at an angle of 30 degrees.

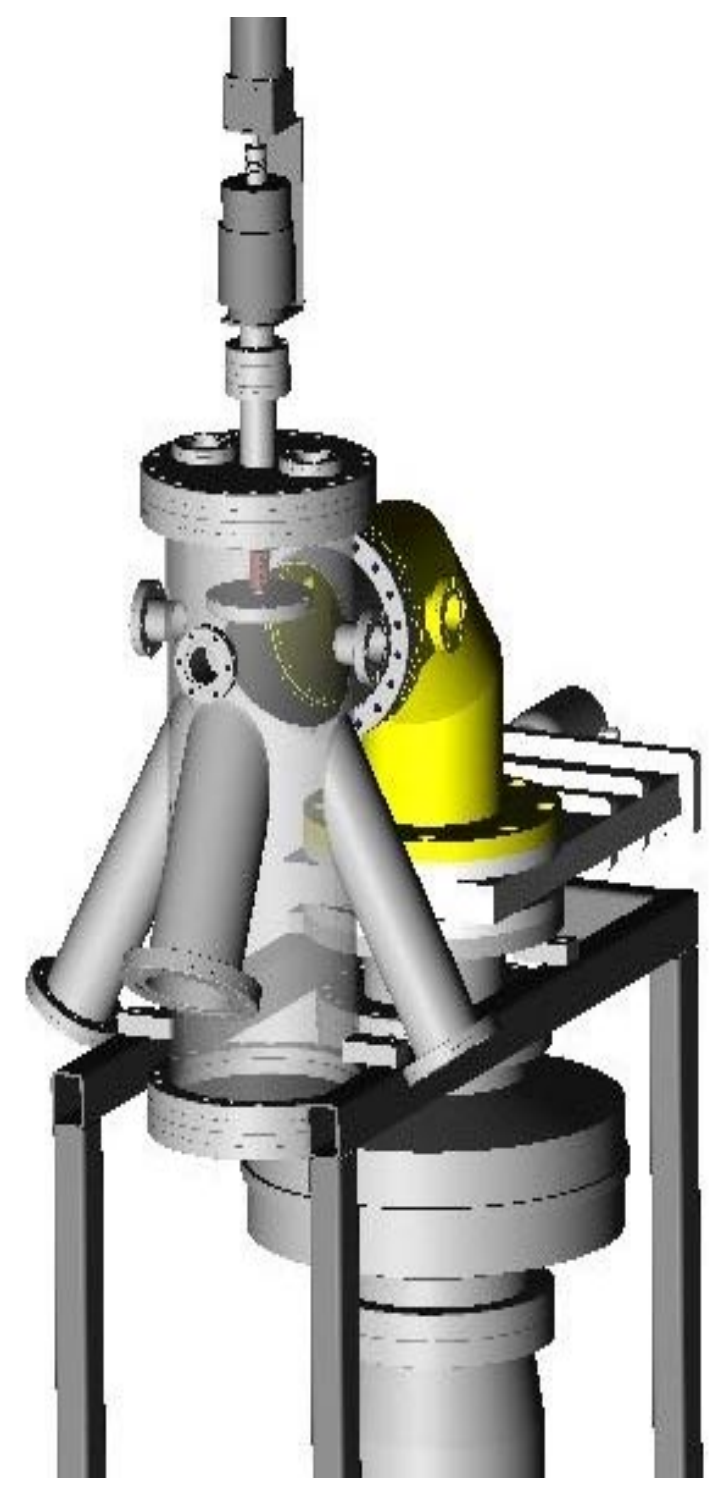

Figure 23. Phase I Experimental Deposition System Design 


\section{Phase I System Features}

- Evaporation sources for liquid precursors

Polysiloxane (silicated hydrocarbon)

Perfluoropolyether (fluorinated hydrocarbon)

Individual QCM deposition monitors

- Kaufman-type ion source

Substrate precleaning

Ion-assist during deposition

Typically run on Argon

- Vacuum system

Stainless steel construction

Electropolished interior and exterior

Ionization and thermocouple vacuum gauges

Oil-diffusion high vacuum pump

Oil-sealed mechanical roughing pump

Base pressure $-10^{-7}$ Torr

- Substrate platform

3" usable diameter

Variable-speed rotation

Thermocouple temperature measurement

Figure 24. Phase I System Features 


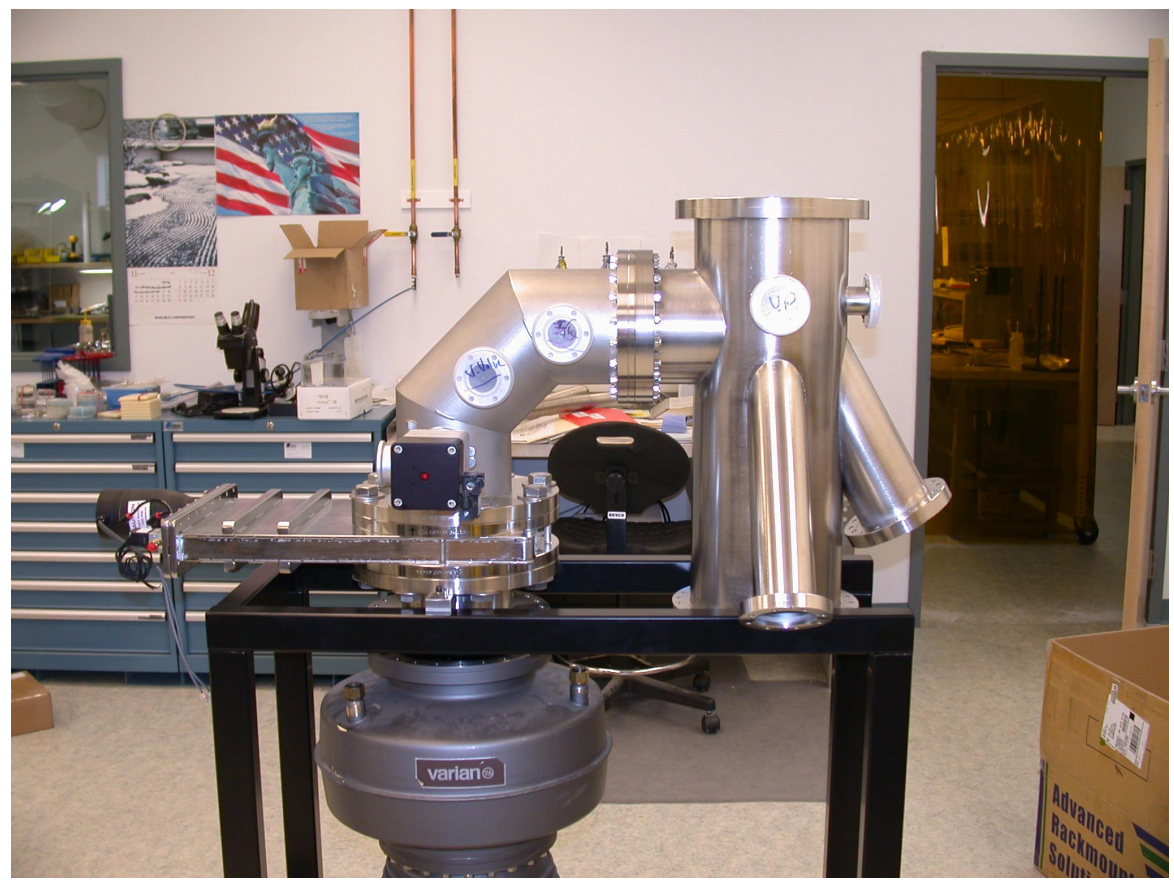

Figure 25. Phase I System Under Construction

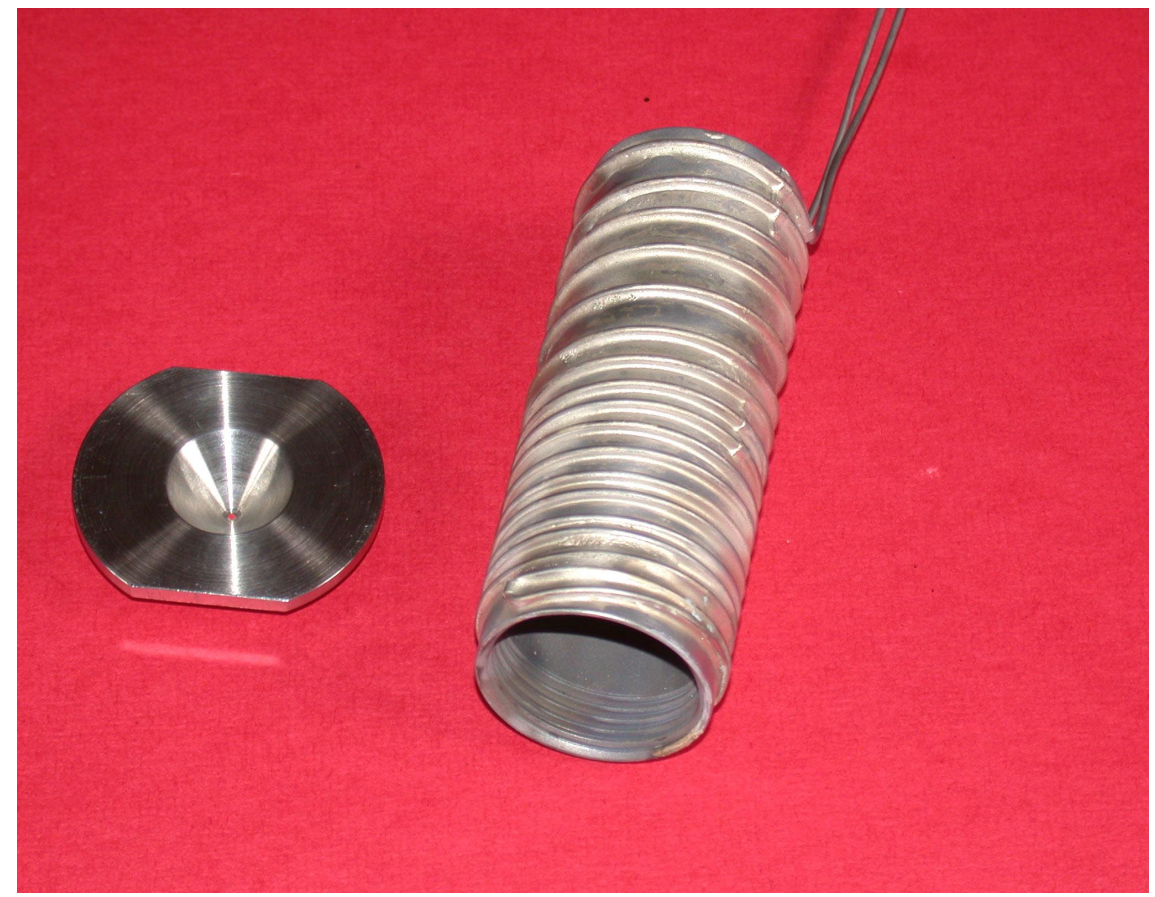

Figure 26. Components of Heated Source for Evaporation of Liquid Coating Precursors 
At PVD Products, DLC coatings were made using a liquid coating precursor polysiloxane (a silicated hydrocarbon) - emitted from one of two independently monitored and controlled flange-mounted evaporation sources (essentially Knudsen-type effusion cells), each with its own quartz crystal microbalance deposition monitor to control coating thickness and deposition rate.

A small (3 cm. beam aperture) Kaufman-type ion source was flange-mounted and aimed at the substrate platform, for both substrate precleaning and ion-assist during deposition. The source was equipped with Tungsten filaments and graphite grids, suitable for operation with inert gases (typically Argon) but not with Oxygen.

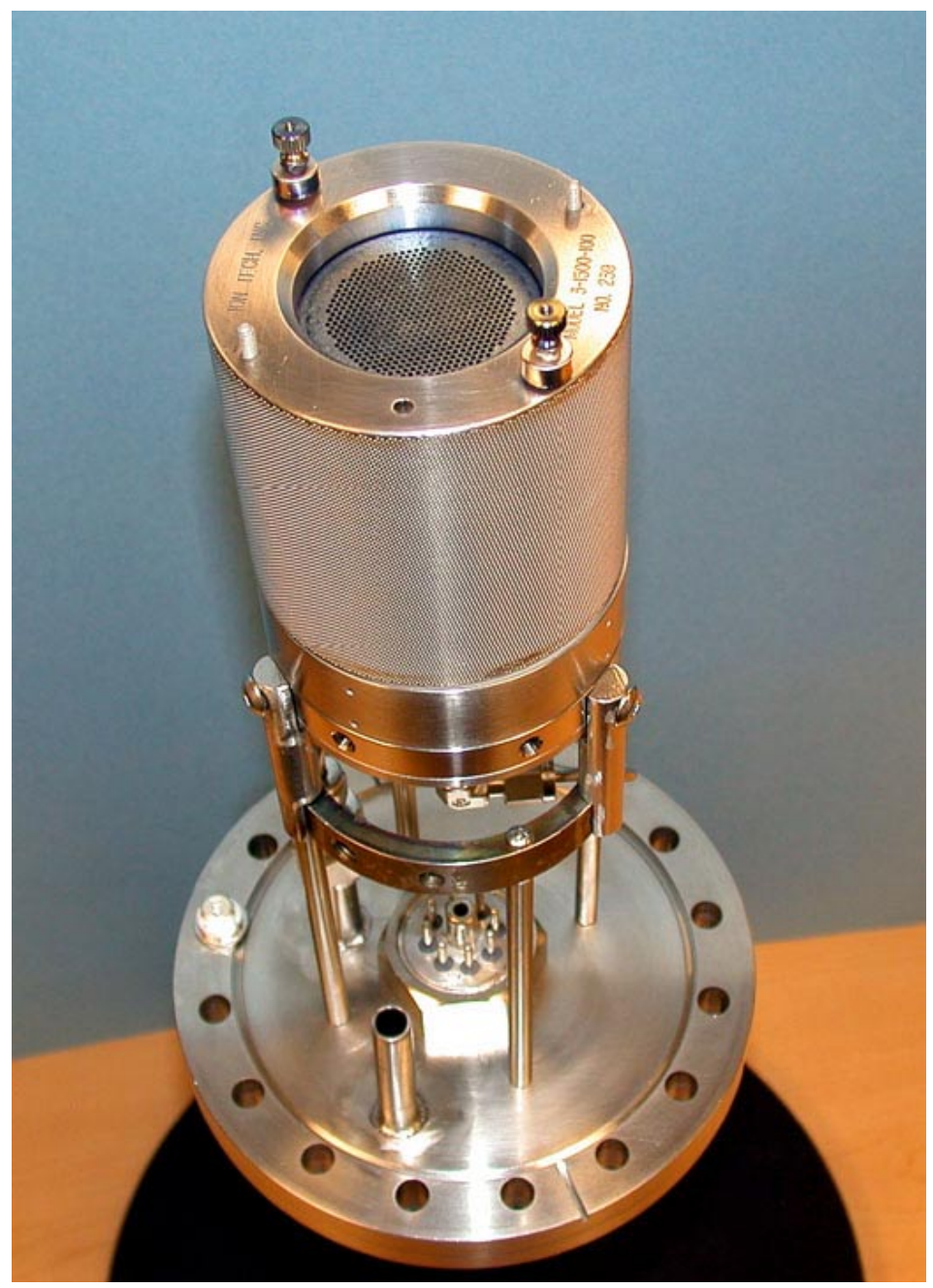

Figure 27. Kaufman-type Ion Source and Mounting Flange (Top View) 


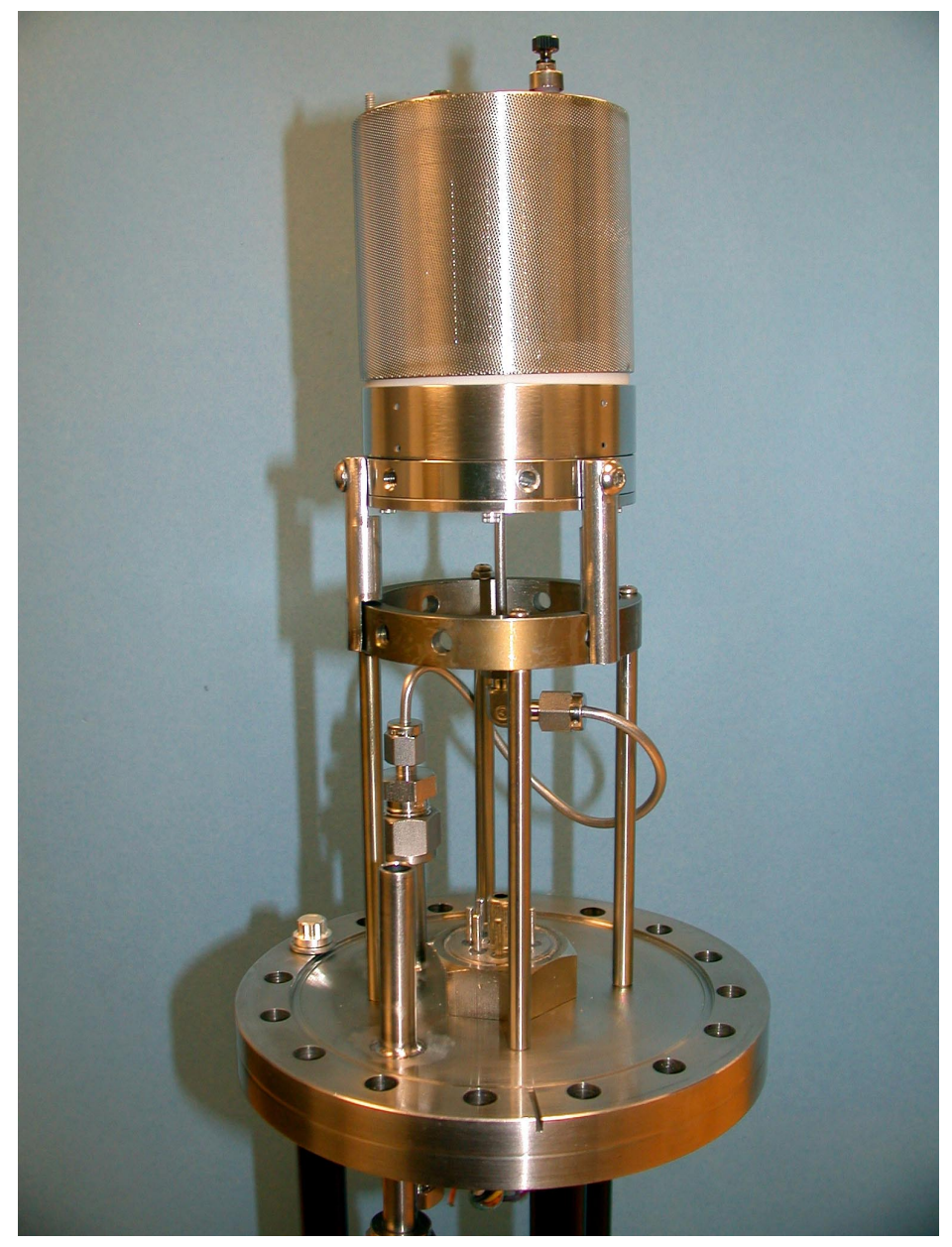

Figure 28. Kaufman-type Ion Source and Mounting Flange (Side View)

The vacuum system featured all-stainless steel construction of the process chamber, which has electropolished interior and exterior surfaces, and a number of small spare ports for future addition of instrumentation. It is equipped with both ionization and thermocouple vacuum gauges. The pumping system consists of an oil-diffusion high vacuum pump, backed by an oil-sealed mechanical roughing pump, and was capable of a base pressure of $10^{-7}$ Torr or less. (In discussions with research groups including the Army Research Laboratory that have done related coatings using polysiloxane diffusion pump oil as a precursor for silicated DLC coatings, it was felt that since the coating process was oil-based, there would be extra expense but no particular advantage in having an oilfree pumping system.) However, if a dielectric coating proves advantageous, it should be deposited in an "all-dry" vacuum system, with an oil-free mechanical roughing pump, and a clean high vacuum pump (either a cryopump or a turbomolecular pump. 
The Phase I system has a relatively small substrate platform ( $\sim 3$ " diameter) due to space constraints. The platform is capable of variable-speed rotation as necessary to improve circumferential coating uniformity, and the approximate measurement of substrate temperature with a rear-mounted thermocouple.

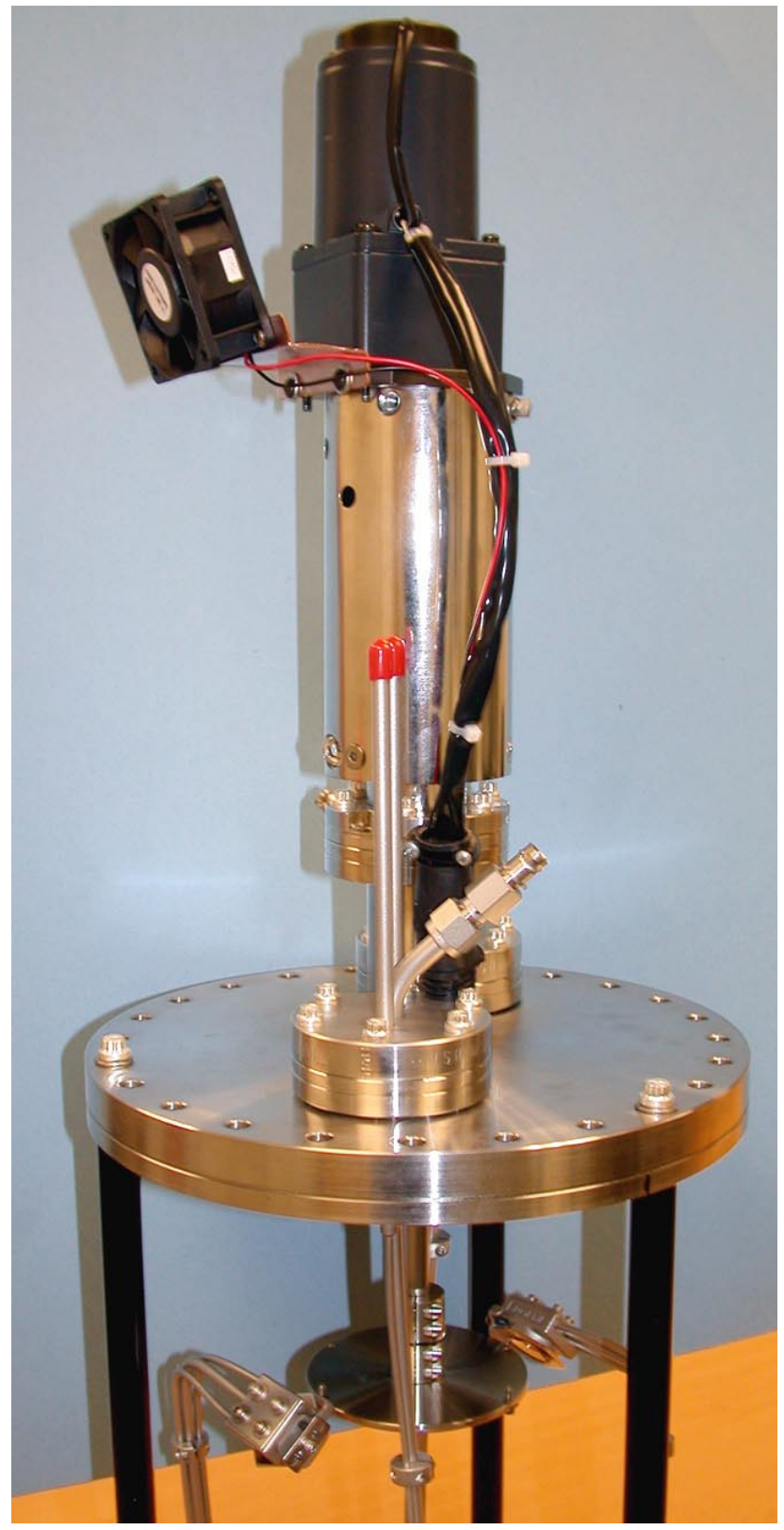

Figure 29. Substrate Platform and Rotary Drive Mechanism 


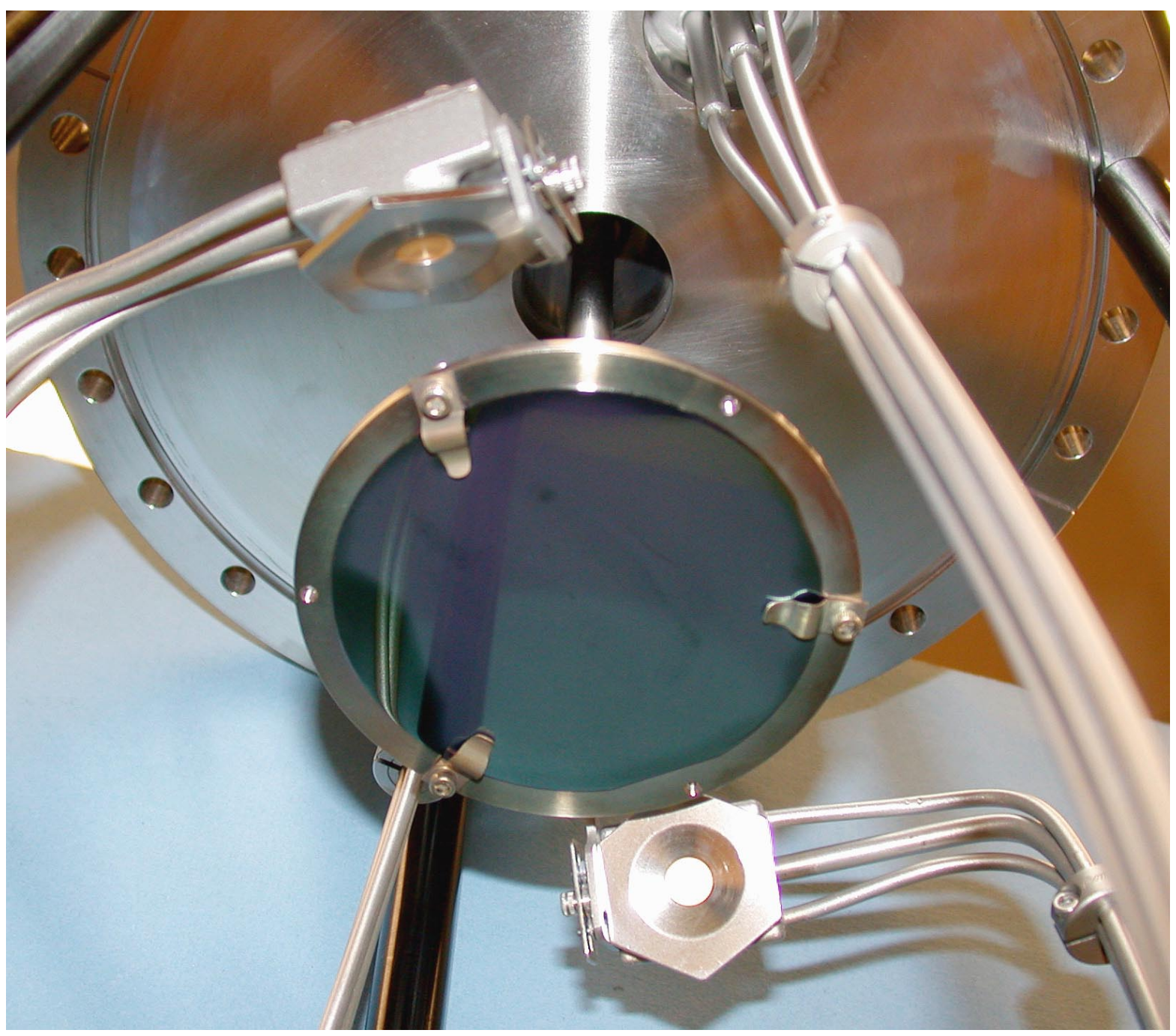

Figure 30. Bottom View of Substrate Platform, showing Quartz Crystal Deposition Monitors 
Coating Deposition and Properties. A series of test coatings of various types of diamondlike carbon ( 1,000 Angstroms thick) were deposited on substrates of fused silica, glass, and Silicon by Southwest Research Institute and PVD Products. These coatings were evaluated by PVD Products, SwRI, and Optical Coating Corporation. The optical and abrasion resistance test data are summarized below, along with a design concept for an IFE optics coating system and recommendations for further work, including a summary of the proposed Phase II program.

The coatings were of three types:

- Undoped DLC, deposited by plasma immersion ion processing (PIIP) at SwRI

- Fluorine-doped DLC, deposited by PIIP at SwRI

- Silicon-doped DLC, deposited by ion beam-assisted deposition (IBAD) at both SwRI and PVD Products

In order to obtain a maximum of results with a minimum of time and expense, the optical transmission and physical durability were demonstrated on small and relatively inexpensive substrates. UV transmission was measured on coatings deposited on small (1") flat fused silica glass $\left(\mathrm{SiO}_{2}\right)$ substrates, fabricated by Opco Laboratory from Corning \#7940 UV Grade material. Small (2") Silicon wafers were also coated, while glass microscope slides were used as witness samples. Although the surface flatness is not critical for Phase I screening tests, the surface finish is important, in determining optical scatter, water contact angle, microstructure and other significant coating properties. Therefore the rms surface roughness of the uncoated substrates was specified at 3 to 5 Angstroms, or less.

Coatings of undoped DLC and silica-doped DLC had both good abrasion resistance and good adherence to Silicon substrates, as tested by Optical Coating Corporation (OCC). However, the fluorinated DLC unfortunately failed both tests. Although none of the coatings had very low $(0.1 \%)$ UV absorption, the undoped DLC had a transmission loss of only $4.3 \%$ at $248.4 \mathrm{~nm}$, with respect to the uncoated substrate. The F-DLC coating loss was $\mathbf{4 6 . 0 \%} \%$, while the Si-DLC coating loss was 78.1\%. At infrared wavelengths from 1 to 5 microns, all DLC coatings had good transmission (within 1-2\% of the uncoated Si wafers). (Note: the water contact angle of the uncoated glass substrate was only $45^{\circ}$.)

\begin{tabular}{|c|c|c|c|c|c|}
\hline Coating Type & $\begin{array}{l}\text { Visual } \\
\text { Color } \\
\end{array}$ & Adherence & $\begin{array}{l}\text { Abrasion } \\
\text { Resistance } \\
\end{array}$ & 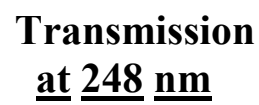 & $\begin{array}{c}\text { Contact } \\
\text { Angle }\end{array}$ \\
\hline DLC & clear & Passed & Passed & $87.1 \%$ & $\sim 75^{\circ}$ \\
\hline F-DLC & brown & Failed & Failed & $42.3 \%$ & $\sim 90^{\circ}$ \\
\hline Si-DLC & blue & Passed & Passed & $10.0 \%$ & $\sim 90^{\circ}$ \\
\hline
\end{tabular}

Figure 31. Summary of DLC Coating Test Data 


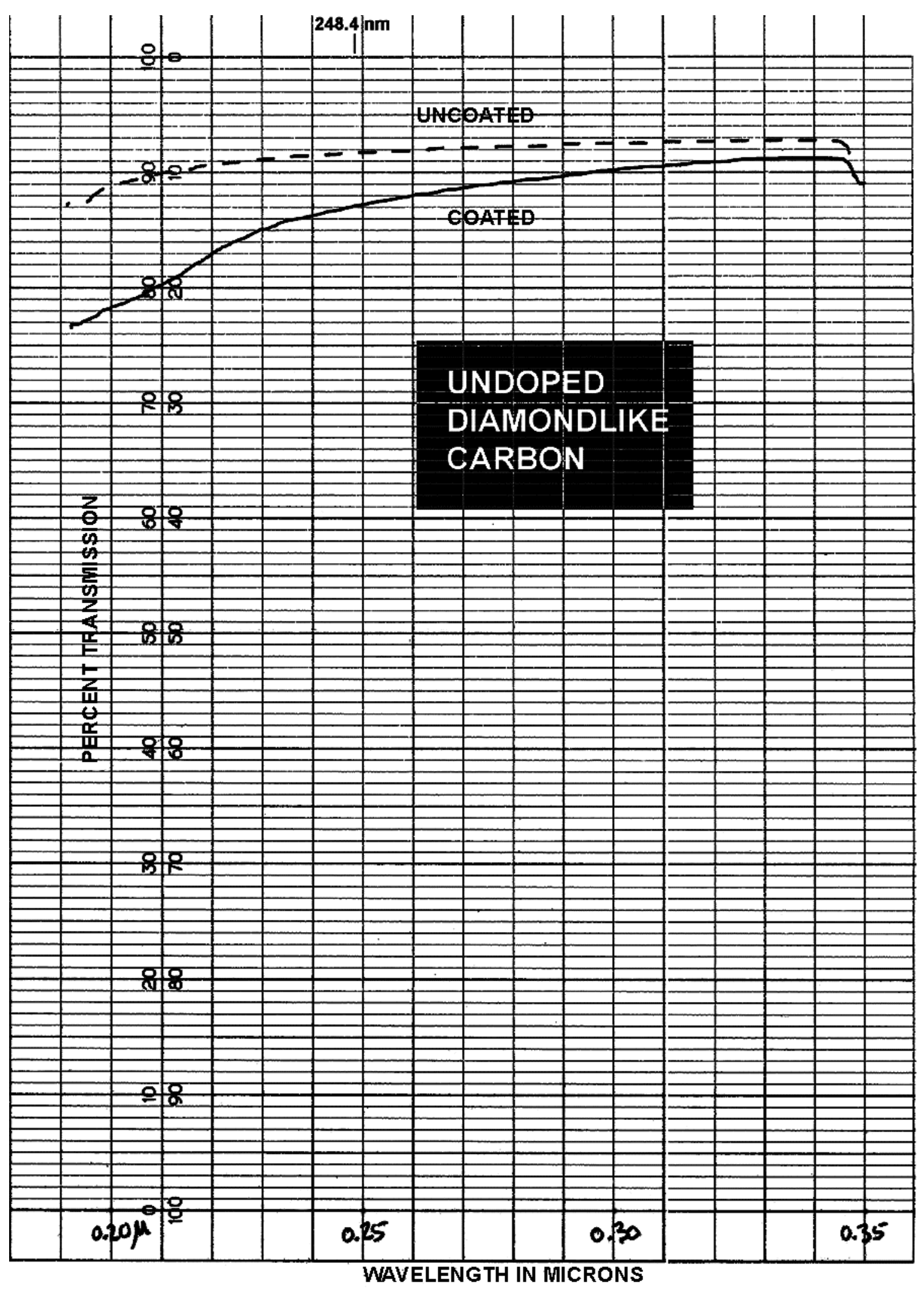

Figure 32. UV Transmission of Diamondlike Carbon (DLC) Coating 


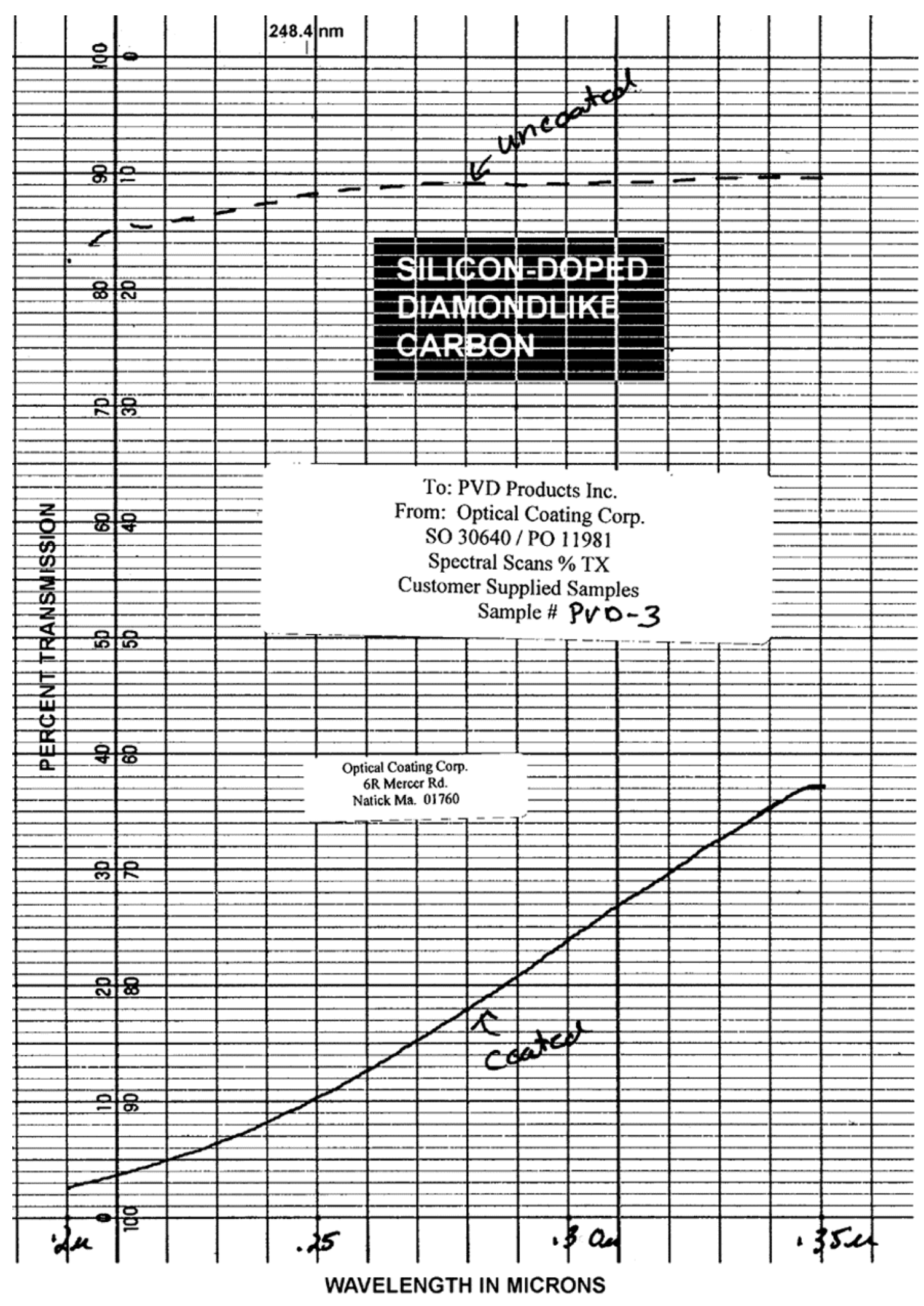

Figure 33. UV Transmission of Silicated DLC Coating 


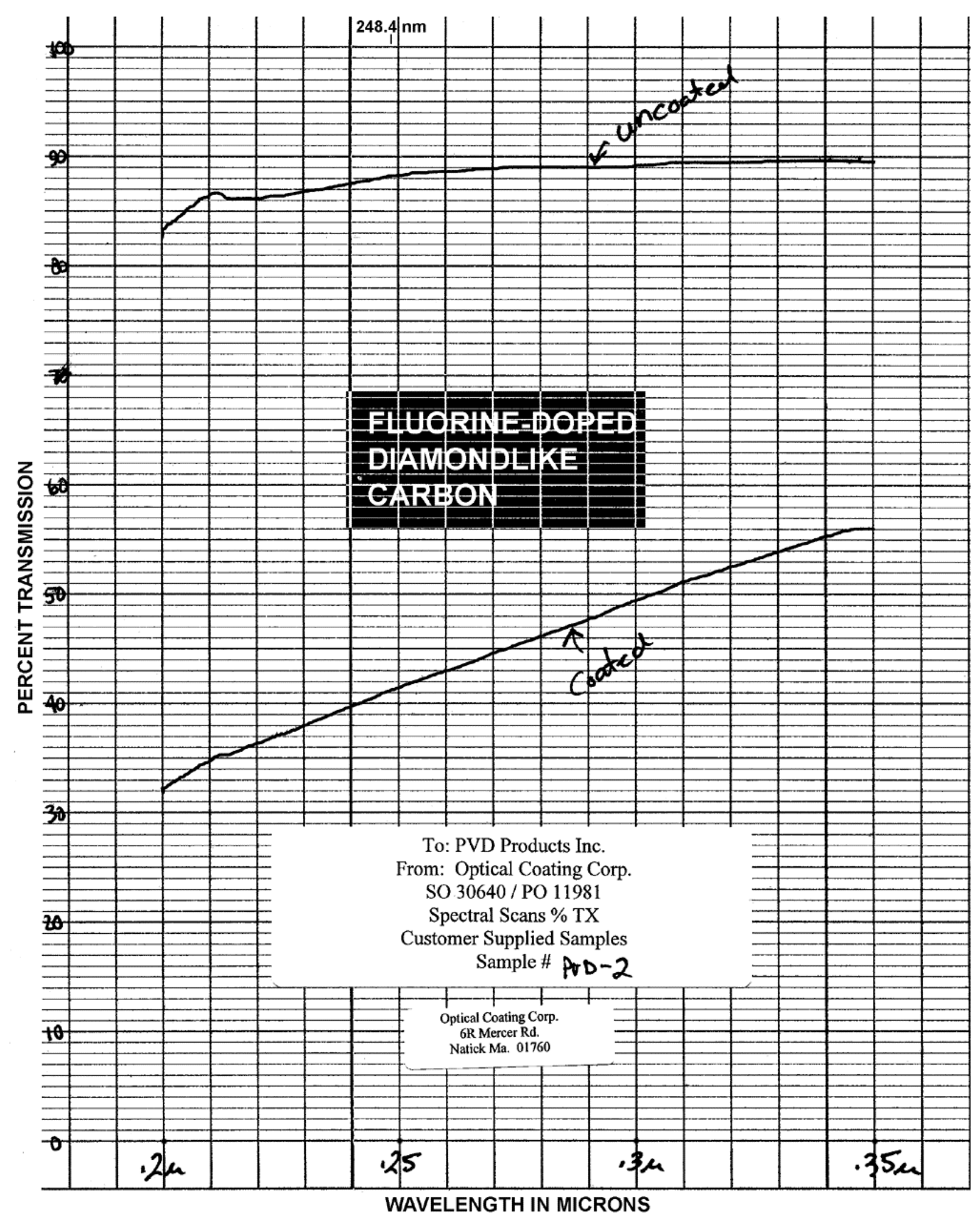

Figure 34. UV Transmission of Fluorinated DLC Coating 


\section{Phase II Deposition System Concept}

The design process for IFE optics coating equipment has two main aspects:

1) applying general principles of contamination control and vacuum engineering applicable to any coating process, including construction material selection and preparation, use of load-locks, types of seals and pumping systems, etc.

2) specific details of the optimum coating process, as determined by analysis of the test results of the Phase I and early Phase II programs, including the type of process, required coating material and/or ion sources, vacuum regime, process parameters, etc.

A conceptual design and preliminary specification for the Phase II prototype coating system is summarized below. The precise details of the actual system that is constructed will be based on the particular process to be selected for further development and implementation in Phase II (for eventual application of reflective and protective coatings on full-scale IFE final optical components in Phase III). The system proposed is flexible, and can be adapted to IBAD, PIIP, sputtering, and/or evaporation processes.

It is highly desirable to incorporate real-time, in-situ contamination monitoring into next-generation IFE optical component processing equipment. A pro-active approach to minimizing optics contamination must be applied to the specification and design of both processes and equipment for the coating, transport, handling, and maintenance of IFE optics.

Regardless of the deposition method or process pressure employed, the coating process chamber must be capable of evacuation to near-ultrahigh vacuum pressures, to thoroughly outgas all internal components and substrates, and remove residual gas contamination before high-purity process gases are admitted to the system. In order to make superior coatings, the vacuum performance of the system must be of near-UHV quality, with a routinely achieved base pressure of no more than $1 \times 10^{-8}$ Torr (using a load-lock design) in 1 hour, when all sources, tooling, and shields have been installed and the substrates are loaded. Furthermore, the system's pumping speed shall be sufficient to keep process pressure at $1 \times 10^{-4}$ Torr or less, for a worst-case total gas load of $150 \mathrm{sccm}$ (if the ion source, neutralizer, and magnetron source are all running simultaneously). 


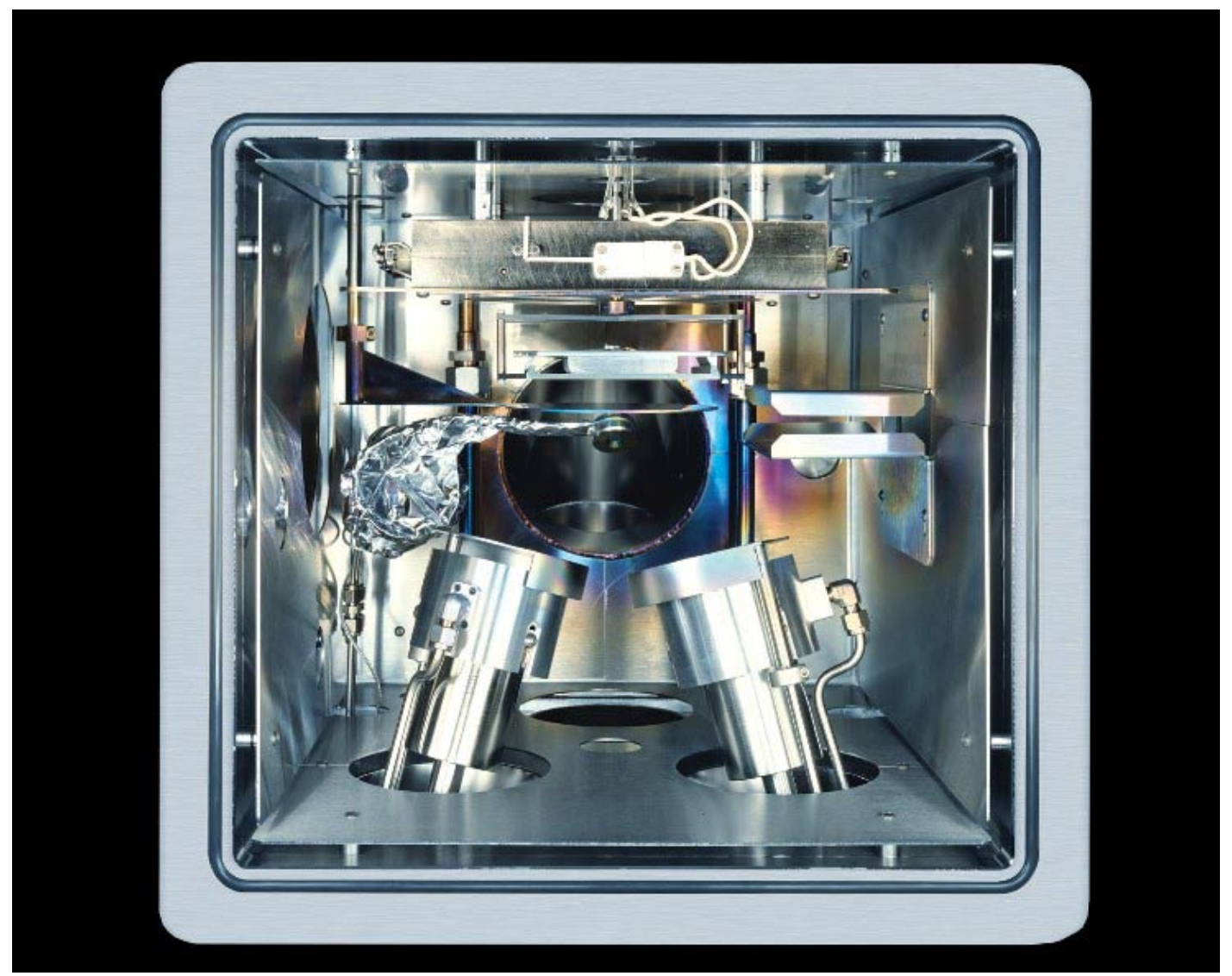

\section{Figure 35. Multi-source magnetron sputter deposition system, designed and built by PVD Products for MIT}

It is anticipated that the Phase II coating system will be a cubical box of type 304 stainless steel, approximately 18" to 20 " in size. It will have four positions for sources of coating material and ions, all aimed at a 6" diameter substrate platform that has its own shutter to protect substrates from contamination. The sources are expected to consist of:

1) A relatively low-energy ( $\sim 20$ to $200 \mathrm{eV})$ filamentless end-Hall ion source (KRI Model EH-400) with hollow cathode neutralizer, for substrate precleaning and potentially for smoothing, ion assist during deposition, coating post-treatment, and potentially for removal of coatings without substrate damage. It will have its own shutter, and be capable of producing an adjustable mixture of Argon and Oxygen ions.

2) Two 4" magnetron sputter sources; one DC-powered for Aluminum deposition, and one powered by RF or anti-arcing pulsed DC for oxide overcoating deposition. Each will have its own shutter.

3) A third coating material source, either another magnetron or a thermal evaporation source, with its own shutter. 
The deposition system depicted in the photograph above has two magnetron sputter deposition sources, and an additional unused source position in the baseplate. In the Phase II system, there will be a fourth source port, on which will be installed a high-current endHall ion source (Kaufman and Robinson, Inc.) equipped with a hollow cathode electron source for beam neutralization, as depicted in Figure 34. Linear versions of this source are also made; they would be suitable for Phase III production coating systems, particularly inline systems.

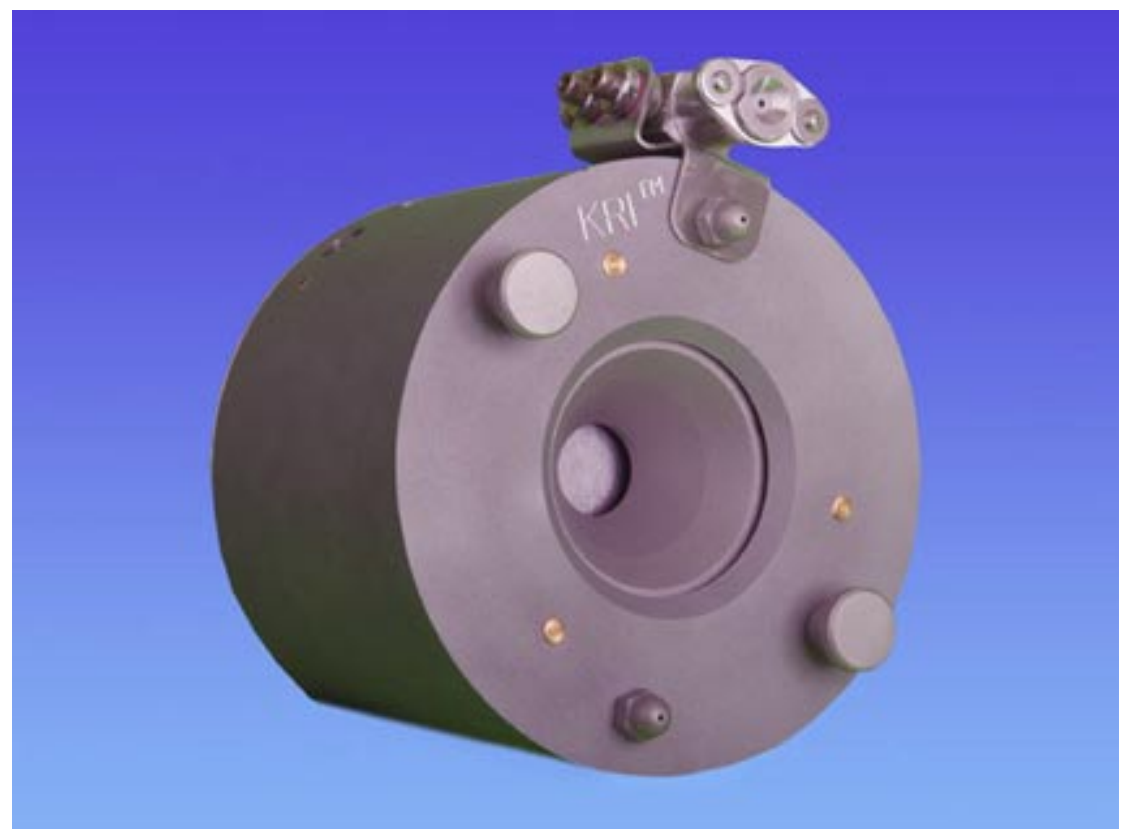

Figure 36. End-Hall Ion Source (Kaufman and Robinson, Inc.)

A summary of the specifications of the Phase II system is given below:

- Two or three 4" magnetron sputter deposition sources

1 DC, 1 RF, 1 TBD (may be evaporation source instead)

Equipped with individual shutters

- End-Hall ion source (KRI Model EH-400 w/ HCES)

Substrate precleaning

Ion-assist during deposition

Typically run on Argon, Oxygen, and/or $\mathrm{Ar}+\mathrm{O}_{2}$

Adjustable gas mixture capability required 
- Movable Faraday cup ion beam current probe

Scan-able along one dimension

Push-pull feedthrough if consistent with base pressure spec

Could also mount on substrate shutter

- Pumping system and vacuum gauging

All-dry pumping system (Turbomolecular high vacuum pump)

Routine base pressure $-1 \times 10^{-8}$ Torr fully loaded

Process gas load up to $150 \mathrm{secm}$

Process pressure $-1 \times 10^{-4}$ Torr maximum at full gas load

Vibration isolation of pumps from process chamber

Soft roughing and venting at all times

Venting/purging with Ar or dry $\mathbf{N}_{2}$

Ionization high vacuum gauge near substrates

2 thermocouple vacuum gauges (chamber and roughing line)

- Vacuum chamber

Box construction, 18" to 20" dimensions

304 stainless steel material

Electropolished interior, fixtures, shields, substrate platter

Load-lock and substrate manipulator

Ports for instrumentation (RGA, optical monitor, pyrometer)

Viewports for sources and substrates

$150^{\circ} \mathrm{C}$ bakeout capability

Leak checking ports

Material selection for low outgassing and particle generation

Removable 304 SS electropolished shields

- Substrate platform

6" usable diameter

Variable-speed rotation (0-40 rpm)

Pyrometer temperature measurement

Shutter necessary to protect substrates from contamination

- Process monitoring instrumentation

Substrate front surface temperature - LWIR pyrometer Pressure

Rough vacuum - 2 TCs, on roughing line and on chamber

High vacuum - Ionization gauge near substrate

Background/contamination - dedicated RGA

Head position near substrates

Must operate during process, @ $10^{-4}-10^{-3}$ Torr

Deposition rate and coating thickness - quartz monitor 
Ion beam current density - movable Faraday cup beam probe

Through push-pull feedthrough,

or attached to substrate shutter

- Gas lines

304 SS material

Electropolished after cutting and bending

Point-of-use particle filters on all gas lines

As near as possible to process chamber

Purgeable without contaminating process chamber

Metal-sealed mass flow controllers

Regulators must be rated for IC processing equipment

Phase II Deposition System Concept. The Phase II deposition system for coating prototype IFE optical components will be capable of coating over an area $15 \mathrm{~cm}(6$ ") in diameter. The design concept for the Phase II deposition system includes a load-lock chamber with substrate manipulator, as well as process diagnostic equipment including an RGA, deposition monitor, ion beam current density probe, and optical pyrometer for noncontact substrate temperature measurements. One or two sputter deposition sources will be included, and possibly one evaporation source. A low-energy, high-current end-Hall ion source will be used for substrate precleaning and ion beam assist during deposition.

Based on experience with this system, Phase III production coating systems will be designed for substrates up to 1 meter long or more if required, representative of actual IFE final mirrors. At this time, it is estimated that a Phase III system for coating $0.25 \mathrm{~m}$ wide $\mathrm{x}$ $1 \mathrm{~m}$ long substrates would have external dimensions of approximately $0.75 \mathrm{~m}$ wide $X 2.5$ to $3 \mathrm{~m}$ long, including the load-lock chamber. The substrate manipulator arm may add about 1 meter to the system length, depending on how it is designed. Rough estimates of the system weight and cost are 10,000 lbs and \$2-3 million, respectively, for a system to coat 1 meter long substrates. In Phase III, such turn-key large-scale production equipment coating equipment will be made available to DoE IFE optics contractors by PVD Products, and commercial applications will also be pursued. 


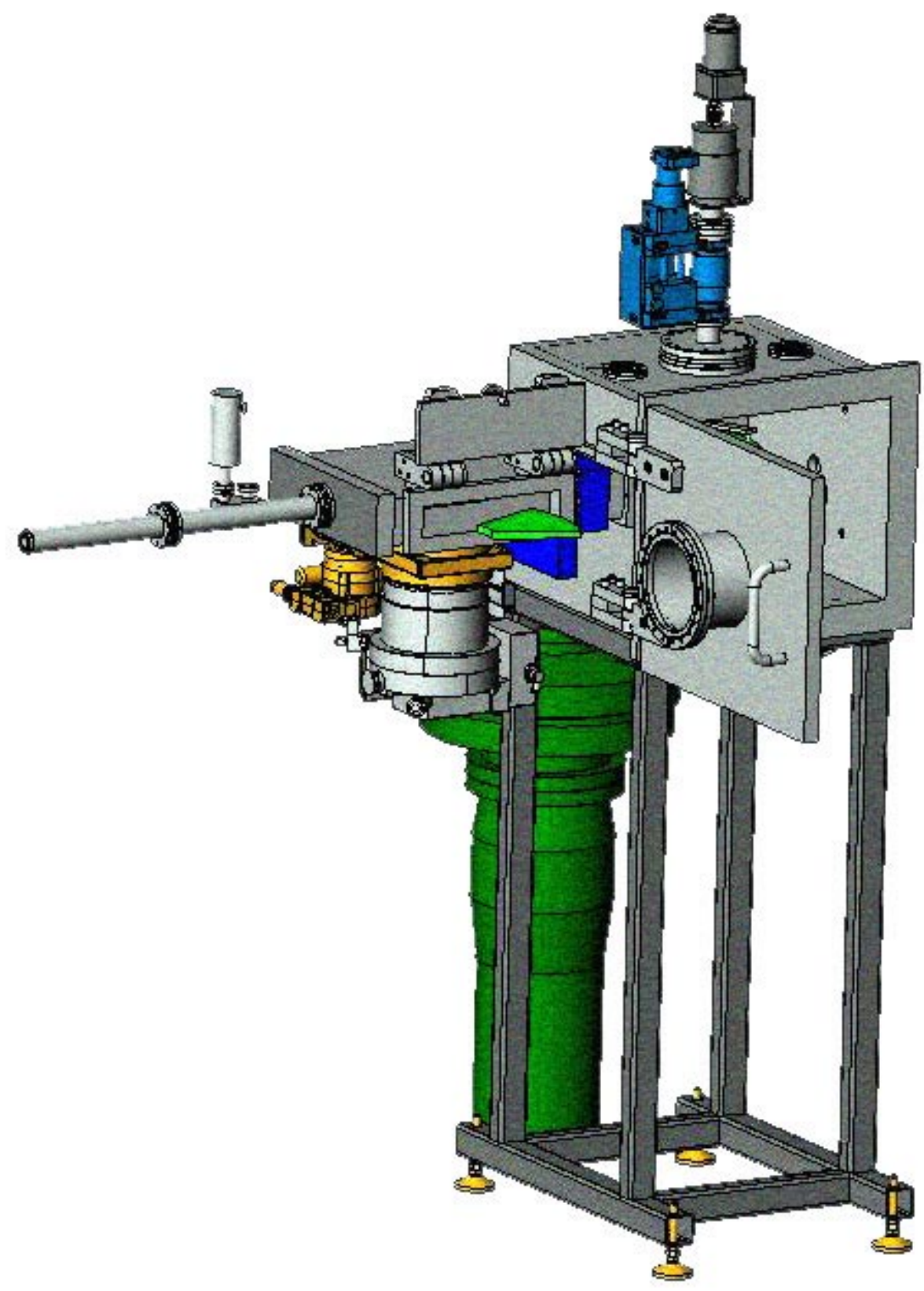

Figure 37. Phase II Deposition System Design Concept

In the design concept drawing above, a diffusion pump is shown as the high-vacuum pump, which would be used on a system for deposition of carbon-containing coatings; for deposition of metals and oxides, however, a cryopump or turbopump evacuated by an oilfree mechanical pump would be employed. In either case, the load-lock chamber would be evacuated by a small turbopump. 


\section{Phase II Deposition System Features}

- Material selection for low outgassing and particle generation

- Substrate and source shutters

- $10^{-8}$ Torr routine base pressure

- Soft roughing and venting

- Dedicated RGA

- IR pyrometer for non-contact substrate temperature measurement

- Load-lock chamber with substrate manipulator

- Ion source for substrate precleaning and ion beam assisted deposition

- Ion beam current density probe

- Quartz crystal coating thickness/deposition rate monitor

- Point-of-use particle filtration of all process gases

- Electropolished system interior and fixtures

- Vibration-isolated pumping system

- Leak-checking ports

- Metal-sealed mass flow controllers for process gases

Figure 38. Phase II Deposition System Features.

The design concept for the Phase II deposition system is illustrated in Figures 37, 39, 40, and 41, showing the load-lock chamber with substrate manipulator, main process chamber, ion and deposition sources, and high-vacuum diffusion pump. Some components have been omitted for clarity, including the mechanical roughing pump, RGA, deposition monitor, ion beam probe, and pyrometer. The exact configuration of sources used depends on which deposition process is pursued in the Phase II program. 
In the front view (Figure 37), the load-lock chamber door is shown open to admit a substrate. The main process chamber will normally be kept at a very high vacuum ( $\leq 10{ }^{-8}$ Torr), while the load-lock pumps down and the substrate and tooling is allowed to outgas. When the pressures in the load-lock and process chambers equalize, the valve between them opens and the substrate is moved into the process chamber by the manipulator. The valve between the load-lock and the process chamber is then closed, and the deposition process begins.

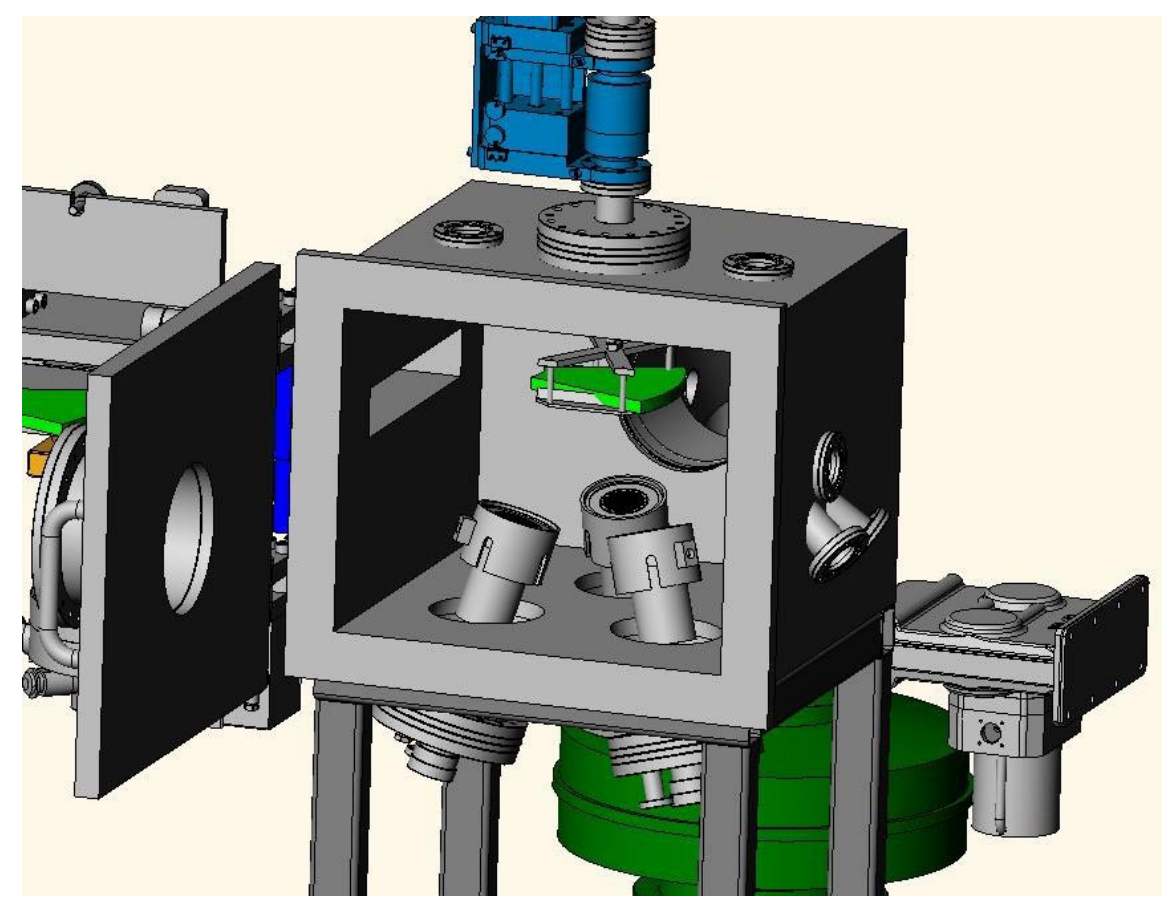

Figure 39. Phase II Deposition System Design - Interior View.

The interior view of the process chamber (Figure 37) shows two magnetron sputter deposition sources, as well as a broad-beam ion source, aimed at the substrate, which is held in a rotary fixture driven by an external motor. In a scaled-up Phase III deposition system for meter-length substrates, a linear ion source and rectangular sputtering sources (approximately half as long as the largest substrate dimension) may be used, so that the entire substrate area will be bombarded by the ion beam and coated by the sputtering sources, as the substrate moves transversely. If designed for PIIP deposition, the chamber interior would resemble the depictions in Figures 16, 18, and 20. 


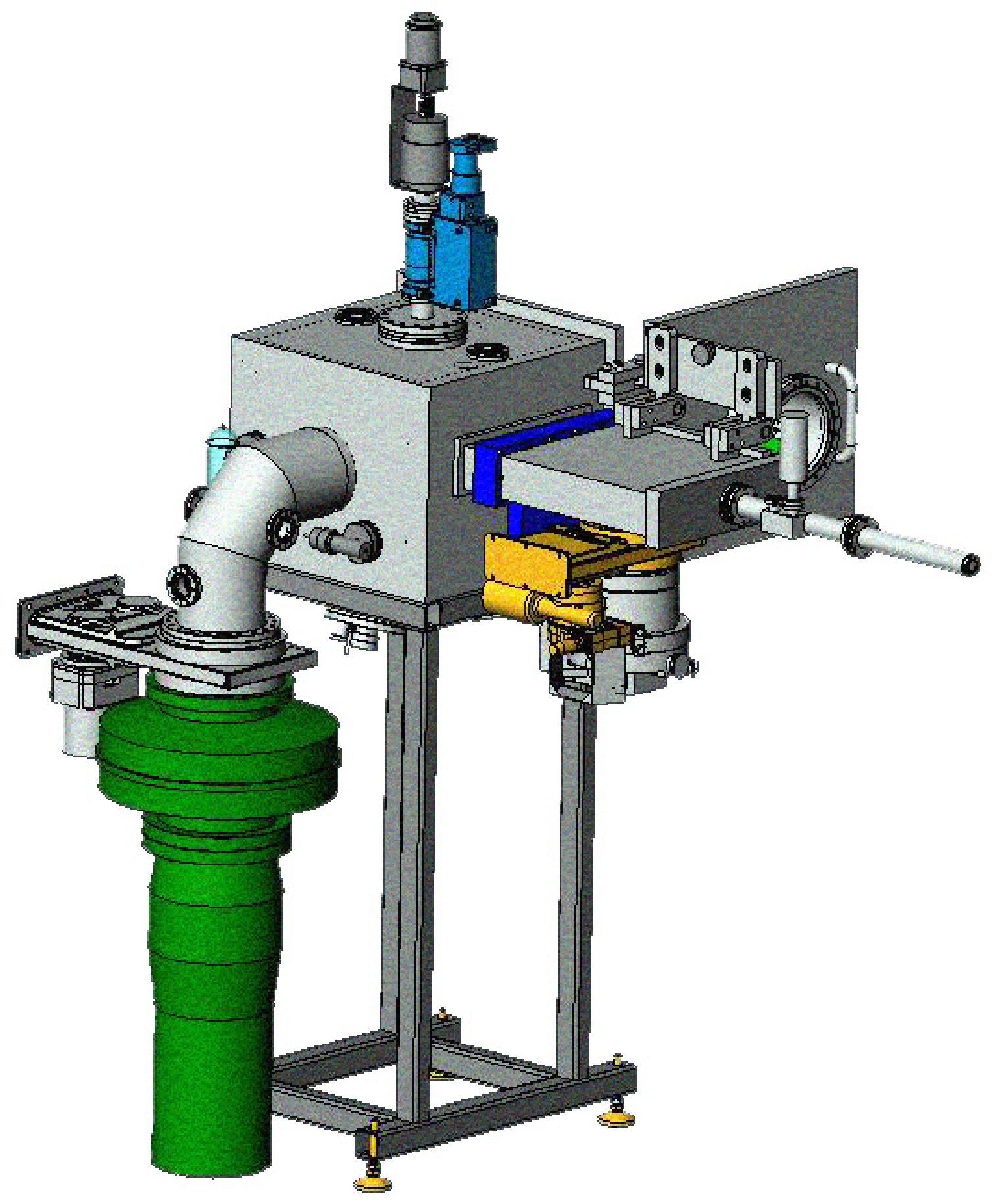

Figure 40. Phase II Deposition System Design - Rear View. 


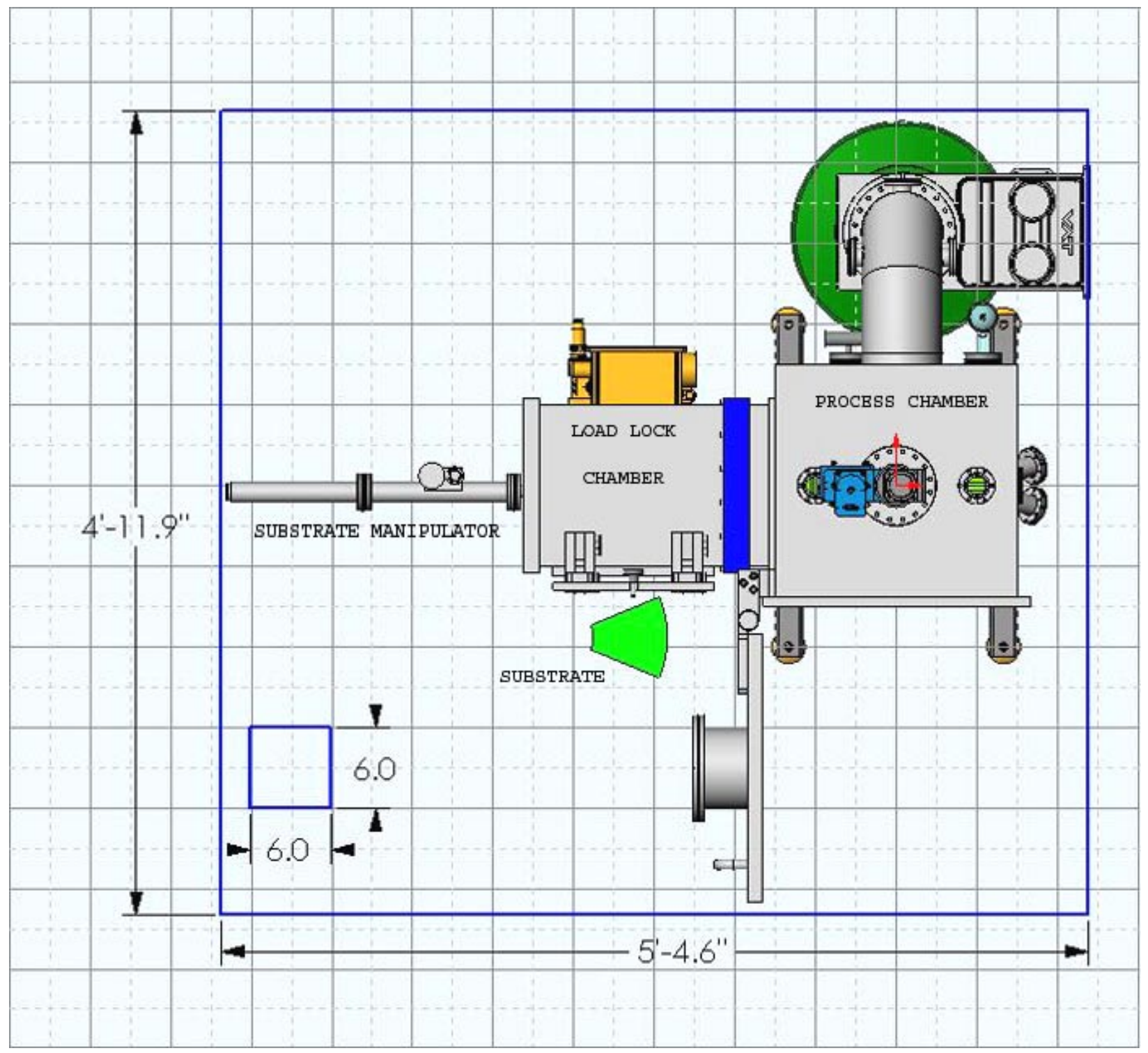

Figure 41. Phase II Deposition System Design - Top View on 6” Grid.

The Phase II system will fit within an area approximately 5 feet by 5-1/2 feet, including the roughing pump, which is not shown. An additional $2-3$ feet of clearance space will be allowed on all sides of the system, to allow access for operation and maintenance. 
Design Philosophy for Phase II Deposition System. The following list of general principles, which will be followed in design and construction of both Phase II prototype and Phase III production coating systems, was generated at PVD Products as part of the Phase I program:

1) Use of only space-qualified materials in construction, which must have very low potential for either outgassing or particle generation.

2) The entire system should be easily cleanable (inside and outside), and have a low potential for trapping contamination (including rounded interior edges and corners wherever possible). Interior walls, shields, fixtures, and tooling should be smooth and electropolished. Removable internal shields are provided to prevent coating deposition on chamber walls.

3) A substrate shutter will be extremely useful in minimizing light absorption and scattering caused by interfacial contamination between the coating and the substrate. It would allow the substrate surface to be protected during operations with the potential to contaminate the substrate, including pumpdown, system plasma cleanup and/or bakeout, warmup of the various sources, interruptions in the process, and post-deposition venting to atmosphere. Even with a substrate shutter, it is still very important to do gradual, "soft" roughing and venting, to avoid depositing particles on the substrate.

4) It is important to minimize both cross-contamination and self-contamination by the various ion and evaporation sources. Each source should have an individually controlled shutter, and be aimed so that its beam is not reflected directly back at itself, or at any of the other sources. This means that the ion beam should not emanate from directly below the substrates, but rather strike the substrates at some angle, in the range from about $\mathbf{1 5}$ to $\mathbf{4 5}$ degrees from perpendicular. These measures taken together will minimize: 1) backsputtering of substrate, fixture, and coating materials and other contaminants back into the ion source, and 2) coating materials bouncing, dropping, evaporating, or dripping into the ion source.

5) The substrate tooling should be designed to be easily cleanable, with minimum potential for trapping contamination. Tooling design should employ removable inserts to hold the substrates in the tooling so they do not require repeated handling during loading, unloading, cleaning, coating, testing, storage, and shipment.

6) Use of a dedicated RGA (residual gas analyzer) to identify contaminants will be necessary to understand how to make a good, repeatable coating, and to troubleshoot the process if a substandard coating result occurs. Provisions should be designed into the system for mounting an RGA head as close as possible to the substrates, and differentially pumping on it to allow operation during the coating process, at pressures up to the mid-10 ${ }^{-4}$ Torr region. 
7) There should be an ion gauge port located to allow pressure measurement as near to the substrates as possible, so the true conditions under which good films are grown can be known.

8) For the same reason, the Phase II system should have the capability to measure and record the temperature of the front surface of a substrate, since temperature is one of the key factors in thin film growth. For the relatively low substrate temperatures envisioned $\left(<150^{\circ} \mathrm{C}\right)$, use of a long-wavelength infrared pyrometer is the best method.

9) The vacuum chamber design geometry and port locations should allow optical monitoring of the coatings to be done (not only to look at coating thickness, but to observe the optical behavior of the coating in vacuum and to help detect surface contamination). If the desired coating or contaminant layer thickness is small (tens of nanometers or less), an in-situ ellipsometer may be useful.

10) The system should be able to regularly attain a base pressure of $1 \times 10^{-8} \mathrm{Torr}$, with substrates, shields, and tooling installed. This will require a separately pumped load-lock chamber, and a mild $\left(150^{\circ} \mathrm{C}\right)$ chamber bakeout capability, with internal heat lamps or external heating tape. The use of a short-wave UV lamp in the load-lock chamber and/or the main process chamber can remove organics and moisture from the surface of the substrate and tooling, without appreciably heating the bulk of the substrate itself.

11) A Faraday cup-type ion beam current density probe is necessary, to relate the arrival rate of the ions at the substrate surface with the flux of the evaporated coating precursor streams. This is required in order to be able to reproduce the best coating results when the process is scaled up. The probe surface must be connected by a shielded cable through an insulated feedthrough to an external microammeter for readout. A small DC power supply is needed to bias the probe surface 20 or 30 volts negative, to repel electrons from the ion source's neutralizer so a correct reading of positive ion current density can be obtained. The probe should be moveable across the ion beam just below the substrate surface, so that ion beam profiles can be obtained.

12) All process gases entering the deposition chamber must pass through final point-of-use particle filters. This includes the gases (Argon or Nitrogen) that are used to vent the chamber to atmospheric pressure at the end of a coating run. Interlocks must prevent the inadvertent venting of the chamber using ambient air, even in manual mode.

13) The system must have a soft roughing and venting capability, to minimize particle generation and transport to the substrates. Interlocks must prevent inadvertent fast roughing or venting, even in manual mode. 
In many respects, the coating quality required for effective IFE optics coatings has already been achieved, albeit on a smaller scale, on a daily basis in the microelectronics fabrication industry. Historically, the optical coating community has lagged behind the microelectronics industry, but there is no fundamental reason that this situation should be allowed to continue. A very useful set of recommendations for design and construction of ultra-clean deposition systems for semiconductor fabrication applications is contained in SEMASPEC \#92051107A-STD (SEMATECH Guide for Contamination Control in Design, Assembly, and Delivery of Semiconductor Manufacturing Equipment), dated 08/21/1992. The purpose of this document is to establish a guide for contamination control in the design, assembly and delivery of ultra-clean equipment for manufacturing devices with submicron feature geometries. The relevant portions of this specification should be followed to the greatest extent economically feasible in the specification, design, construction, installation, and operation of the Phase II and Phase III coating systems.

Following is a summary of the most germane parts of the SEMATECH specification, as it applies to equipment for deposition of low-maintenance protective coatings for IFE final optics, particularly from the standpoint of contamination control:

\section{Relevant Excerpts Adapted from SEMASPEC \#92051107A-STD:}

General. Deposition systems must not significantly contaminate either the product or the surrounding clean room. The system shall minimize the generation of particles and vapors by the system itself, limit the redistribution of these contaminants within the system to the product, and protect the system and the product from external contamination sources (Sec. 1.1). Reduction of the generation of particles (and contaminant vapors) is of primary importance, followed by the reduction of their transport to the substrates (Sec. 4.6). System construction materials exposed to the product shall be selected for low outgassing, and suitability for use at pressures of 1x10-8 Torr, or less (Sec. 4.8.2.1). Chambers shall be made from type 316L low-carbon stainless steel; after machining, welding, and leak testing, interior surfaces shall be mechanically polished to a 32 microinch finish, then electropolished to a 10 microinch finish (Sec. 4.8.2.2).

Equipment Surfaces. Exterior surfaces shall be either stainless steel, painted steel, or anodized aluminum, shall be corrosion resistant and cleanable, and shall resist particulate generation and outgassing. Interior surfaces to which the product will be exposed shall be of electropolished type 316L stainless steel (10 microinch finish). All surfaces should not generate vapors or particles, nor be adversely affected by repeated wiping (Secs. 4.2.2, 4.6.1.1, 4.6.1.2, and 4.7.2). Surface finish shall be smooth, in order to minimize generation of particles and adsorption of contamination, and to facilitate cleaning for preventive maintenance (Sec. 4.7.2). Surfaces shall be cleanable, resistant to water-based and organic solvent-based cleaners, and polished where possible. Interior corners shall not be sharp, but rather designed with generous fillets and radii of curvature (Secs. 4.6.6.1 and 4.7.2). 
System Assembly and Testing. Assembly, testing, and preparation for shipment shall take place in a Class 100 air quality (or better) area equipped with an exhaust to remove any fumes generated by the equipment, and with a cleanroom vacuum (Sec. 5.1.2.1). Assembly procedures shall minimize contamination of the system, including vacuuming simultaneously with all drilling, soldering, and other small tool operations (Sec. 5.1.2.2).

Particle and Vapor Generation. To minimize abrasive generation of particles from objects rubbing against one another, system design shall avoid rapid and/or heavily loaded motions, and impacts. Steel surfaces which must be in rubbing contact shall be coated with Titanium Nitride or Titanium Carbide (Sec. 4.6.3.1). Substrate holding mechanisms shall neither hold parts so tightly as to abrade or chip particles away, nor so loosely that there is relative motion between the substrates and the holders which can create particles (Sec. 4.9.3). Heating elements may flex and spall due to thermal cycling, and shall not be located where they can shed particles on the product (Sec. 4.6.3.3).

Vibration. System vibration should barely be noticeable, so that particles are not dislodged from system components, chamber walls, process carriers, and other internal surfaces (Sec. 4.6.3.6), nor from the cleanroom walls (Sec. 4.2.6). Process chambers must be vibration-isolated from the pumping system, particularly from the roughing pump which should be installed remotely in a service area (Secs. 4.3.1.6, 4.4.1.5, 4.7.5.1, and 4.8.1.6).

Electrostatic Charging. Systems should be designed to prevent electrostatic charge buildup; all metal surfaces shall be grounded (Sec. 4.6.3.7 and 4.7.1).

Contamination Monitoring. System designs shall include in-situ process contamination instrumentation (Sec. 4.7.4), including in-line and/or in situ particle counters and other impurity analyzers. Filtration and particle detection for incoming gases at point of use is strongly recommended (Secs. 4.3.1.3 and 4.4.1.4). The rough pumping line should incorporate an in situ vacuum particle monitoring system. The process chamber shall be equipped with a residual gas analyzer (RGA) to identify gaseous contaminants and leaks (Sec. 4.8.1.1).

Chamber Design. Chamber volume shall be minimized (Sec. 4.7.1). Systems shall be capable of bakeout to at least $125^{\circ} \mathrm{C} / 257^{\circ} \mathrm{F}$ (Sec. 4.8.1.2). Crosscontamination affecting the process shall be minimized between the various parts of the tool (Sec. 4.7.3.1). Moving parts are potential particle sources, and shall be minimized or enclosed where feasible (Sec. 4.7.3.2). Sampling ports shall be included for leak checking, as well as testing for particulate or vapor contaminants (Sec. 4.3.1.5).

The chamber design and process should allow internal cleanup of the deposition system without leaving residual contaminants and while minimizing system downtime (Sec. 4.7.6.1). Subsequent to any chamber cleaning operation, at least 3 
fast pumpdown/venting cycles with ultra-high purity, dry, point-of-use-filtered Argon (or Nitrogen) should be done in a manner that encourages turbulent flow at the chamber inlet and outlet (Sec. 4.8.4.4).

Low-temperature lines and components such as liquid Nitrogen traps should be externally insulated to prevent condensation (Sec. 4.3.1.6).

Blind holes, crevices, deep slots, sharp corners, and enclosed areas that may trap particles, make cleaning difficult, or constitute virtual leaks shall be eliminated (Sec. 4.6.6.1, 4.7.6.1, and 4.8).

Use of elastomer O-rings should be limited (Sec. 4.6.3.2). If used, they shall be free of chemical additives (Sec. 4.4.3.3).

Provision for Leak Detection. Systems shall have a permanently installed manual shutoff valve at the mechanical pump inlet, downstream of a test tee having a leak detector connection valve, to allow leak detection of a fully assembled, operating deposition system (Sec. 4.8.4.5).

Welding. All welded joints shall be automatically orbital, butt-welded in a cleanroom environment using high-purity inert purge gases; avoid socket welds and saddle welds. Internal welds must not exhibit any discoloration or bluing (Secs. 4.3.1.1 and 4.3.4.2).

Vacuum Valves. Valves shall be selected for smooth, slow travel, as well as low leakage, low outgassing, low particle generation, and high cycle life, and shall be of either packless metal diaphragm or metal bellows design. Electronic check valves shall be used, rather than spring-loaded check valves (Secs. 4.3.3.1 and 4.8.3.3).

Gas Lines. The purity of incoming gases must not be degraded by the gas lines or internal plumbing of the system (Sec. 4.3). Mass flow controllers shall be all metal-sealed and selected for low particle generation and outgassing (Sec. 4.3.3.4). Pressure regulators should meet the low particle generation, low outgassing requirements of SEMATECH test method 90210392A-STD. Gas line tubing shall be made of type 316L low-carbon stainless steel. Tubing sections must be cut, faced, deburred, and bent (if bending is required) in a Class 10 air quality environment (Sec. 4.3.4.1). The last step in preparation of tubing (after bending) shall be electropolishing to a 10 microinch finish (Sec. 4.5.4).

Gas Line Filtration and Purging. Final point-of-use particle filters shall be rated at 0.01 microns, and shall be selected for both low outgassing and high particle retention (Sec. 4.3.3.2). Filters shall be located downstream of pressure regulators, as close as possible to the process chamber. Filters shall be followed by an isolation valve, unless the system is equipped with a load-lock antechamber (Sec. 4.3.1.3). Gas line designs shall allow thorough purging upstream and downstream of removable filters (and any other removable components). All gas loops shall be purgeable by a vacuum pump (Sec. 4.3.1.4). 
Slow Pumpdown and Venting. Valves shall be opened and closed slowly during initial stages of both pumpdown and venting, to minimize particle dislodging and redistribution by turbulent gas flow, and to minimize vapor condensation. Venting shall be done with ultra-high purity, dry, point-of-use-filtered Argon (or Nitrogen) at a gas pressure of no more than 3 psig static, and must not be done using any portion of the roughing line (Secs. 4.8.4.1 and 4.8.4.2).

Processing Environment. For optimum coating results with a minimum of product contamination, the air quality (particle count) in the cleanroom where the system is installed should be Class 1 (per FED-STD-209 for Clean Room Environments). Real-time, in situ monitors for both particulate and gaseous contamination should be installed in the same area the system is located (Sec. 4.6.5.1). Ambient temperature should be 67 to $69^{\circ} \mathrm{F}$, and humidity should be between $37.5 \%$ and $42.5 \%$ (Sec. 4.1 .1 ).

Service chases should have Class 100 or better air quality (Sec. 4.1.2). Process bay ambient air pressure should exceed service chase air pressure, by up to 0.038 mm Hg or 0.02" $\mathrm{H}_{2} \mathrm{O}$ (Sec. 4.2.1), even during maintenance operations. System components requiring frequent maintenance or cleaning (including filters and mass flow controllers) shall be accessible from the service chase side (Sec. 4.3.1.5). CRTs should not be located in the process area, due to the electrostatic accumulation of particles on the display face (Sec. 4.2 .7$)$. 


\section{$\underline{\text { Applications }}$}

The availability of robust, protective packaging methods for coated precision optical components will have many benefits in addition to enabling IFE research and development of practical fusion energy systems. Potential applications areas include precision optical components for satellite systems, laser material processing systems, and optical measurement instruments; packaging operations in the production of optoelectronic devices; and also for LIDAR, remote sensing, and other aerospace imaging systems. Both the ophthalmic and display industries could greatly benefit from a durable, cleanable, contamination-resistant transparent coating. Many non-optical commercial applications as well will be found in the manufacture of data storage, microelectronic, and microelectromechanical (MEMS) devices, as well as many types of mechanisms (bearings, motors, etc.) requiring wear and friction reducing coatings.

The principal benefit offered by successful optics-protective coatings is the mitigation of handling risks for costly precision optics, not only for IFE systems, but also for:

- Tactical and battlefield lasers

- Surveillance and remote sensing satellites

- Solar cells for space power systems

- Ground and space-based telescopes

- Atmospheric LIDAR systems

- Space optical communications

- Inertial confinement (laser-induced) fusion research and weapons simulation

- Free-electron-laser development

- Aircraft canopies and cockpit displays

- Imaging systems of all types, including optical inspection and machine vision systems

- Displays and projection systems of all types

- Optoelectronic component manufacture

Figure 42. Potential Government and Commercial Applications 
DoE Applications. The first priority of this SBIR program is to fulfill the needs of the DoE for durable, low-maintenance protective coatings for full-scale IFE final optics components. This technology offers enormous potential benefits to the DoE, in the areas of IFE optics maintenance cost reduction, improved component reliability and performance, and enhanced practicality of laser-driven IFE systems. Demonstration of a "mid-scale" coating system during Phase II will also provide a solid foundation for future scaleup of the technology to a coating system for meter-length or longer substrates in Phase III. Coated samples will be provided early in Phase II to UCSD, IFE optics contractors, and cognizant DoE laboratories for testing and evaluation. Furthermore, the requirements of contractors for ultraclean deposition systems for IFE optics coatings will be determined and addressed

Other promising potential DoE applications include optics for fusion plasma diagnostic instrument optics and EUV/soft X-ray lasers, and reflective masks and projection optics for Extreme Ultraviolet Lithography (EUVL) systems and light sources.

Military Applications. If the needed optical component packaging technology can be developed for IFE optics, then low-maintenance protective coatings will become available for HEL optics for missile defense and tactical laser systems, facilitating important DoD programs including ABL (AirBorne Laser), GBL (Ground Based Laser), SBL (Space Based Laser), ATL (Advanced Tactical Laser), THEL (Tactical High Energy Laser), SolidState Heat-Capacity Laser (SSHCL), and High Average Power Laser (HAPL).

There are several other promising potential military applications, including critical optics for surveillance satellites, antireflection coatings for optical bulkheads for high power laser systems, flexible protective coatings for large deformable and deployable mirrors for space-based telescopes, and durable release coatings for optically polished, reusable replication mandrels for large mirror fabrication.

Protective/antireflective coatings are needed for making practical use of less durable (softer and/or more hygroscopic) optical materials, including alkaline-earth and transition metal fluorides for UV applications, and delicate IR materials including $\mathrm{ZnS}$, ZnSe, and heavy metal fluorides, and detector materials including GaAs and HgCdTe.

Also useful would be durable, low-maintenance transparent protective coatings for aircraft canopies and cockpit displays.

Important non-optical military (and NASA) applications include very high-reliability tribological coatings for spacecraft mechanisms (which have been a significant problem in the past, e. g. the failure of main antenna deployment on the Magellan Jupiter probe), allowing increased confidence levels in the remote deployment of communications antennas and other critical subsystems for space probes, communications satellites, and surveillance systems.

Coating-based anti-biofouling surface treatments would be very useful for many Navy applications, if they have sufficient durability when exposed to salt water. 
Other Government Applications. Non-military Government optical applications include, atmospheric LIDAR for detection of pollution and clear air turbulence, free-space optical communications, free-electron-laser development, and support of the continued progress in short-wavelength lithography and nanofabrication.

Commercial Applications Development. Coatings suitable for the protection of the surfaces of IFE optical components should also have several commercial applications, including the markets of precision optics, ophthalmic optics, data storage, MEMS, and other optical (as well as tribological) applications.

Private-sector uses for such packaging coatings will be found in optics for high-power laser material processing, displays, ground- and space-based astronomical optics, and ophthalmic lenses, as well as low-maintenance architectural (window) coatings. Key nonoptical commercial applications are likely to include packaging coatings for integrated circuit and optoelectronic device fabrication, as well as in surface protection of magnetic and optical recording heads and storage media (6), the manufacture of thermal ink-jet printer heads, and the fabrication of microelectromechanical systems (MEMS) and microoptoelectromechanical systems (MOEMS).

A key aspect of Phase II commercialization activities is determination of the potential market for PVD Products to provide both deposition equipment and commercial coating services, beginning in late Phase II and continuing into Phase III commercialization.

Each application will be addressed in Phase II by determining what laboratories and optics contractors are actively involved in each applications area, and arranging to provide major potential customers in both optical and non-optical applications areas with sample coatings for their application-related testing and evaluation. For those applications for which the test coatings are successful, specifications and price quotations for the appropriate deposition systems and/or coating services will be drawn up by PVD Products, in consultation with each potential customer.

Contact will be established with representative customers in each market area, to determine the requirements of each application, the potential market, the types of components to be coated, and what performance and environmental tests they would have to pass. Potential customers in all will be contacted, and a business and technical plan will be drawn up and executed to provide sample coatings in Phase II for evaluation by key potential customers in application-related testing. Based on the results of these activities, a detailed marketing plan will be drawn up for the Phase III program. 


\section{Conclusions and Recommendations}

Summary. The original direction of the Phase I program was to apply fluorinated diamondlike carbon coatings to laser beam turning and focusing mirrors, to which a reflective coating had previously been applied. This required that the DLC overcoating have low optical absorption at the KrF laser wavelength of 248 nanometers. In the course of the activities of this program and discussions with the DoE Technical monitor and other researchers in the High Average Power Laser (HAPL) program it became clear that:

- the reflective coating of choice is Aluminum, although what the best thickness and deposition method are remain to be determined

- the substrate material will probably be superpolished Silicon Carbide

- the utility of a hard protective coating with low optical absorption has become clear

- fluorinated DLC was not durable, which seems to be a limitation of the material itself

- fluorinated DLC was not adherent, because the bonding layer normally employed in non-optical applications could not be used because it was not transparent

- undoped DLC coatings deposited by PIIP showed good durability and reasonably high transmission at $248.4 \mathrm{~nm}$, and should be further explored if $\mathrm{Al}_{2} \mathrm{O}_{3}$ or $\mathrm{SiO}_{2}$ do not prove to be suitable overcoating materials; DLC's relatively high contact angle for water vapor will tend to reduce optics contamination

- the overcoating material must be one with lower optical absorption than the DLC coatings deposited in the Phase I program, with $0.1 \%$ loss as a goal

- the most promising candidates for IFE optics protective coatings other than DLC are the hard, high-bandgap materials Aluminum Oxide $\left(\mathrm{Al}_{2} \mathrm{O}_{3}\right)$ and Silicon Dioxide $\left(\mathrm{SiO}_{2}\right)$; however, they have considerably lower water contact angles than DLC, and will probably accumulate more water vapor-borne contamination

- the principles of contamination control and process equipment design developed in the semiconductor industry must be applied to make the best possible coatings for IFE optical components

- load-lock chambers should be used, to isolate coating process chambers from as much contamination as possible, and keep them at near-UHV pressures except when maintenance operations are required

- optical coatings should be deposited on IFE optical components in process chambers that have been evacuated to near-ultrahigh vacuum $\left(<10^{-8}\right.$ Torr $)$ 
- the reflective coating and transparent overcoating should be applied in the same vacuum process cycle, to minimize interfacial contamination and defects

- to minimize growth of large, rough grains in the Aluminum coatings, as well as the possible distortion of the very precisely fabricated and costly IFE final optics substrates, any coating process will probably have to keep the substrate temperature less than or equal to $150^{\circ} \mathrm{C}$. In the Phase II deposition system to be constructed at PVD Products, the substrate temperature will be measured directly, with a long-wavelength infrared pyrometer

- ion sources, if used, should be of the water-cooled end-Hall type, to minimize excessive heating which may roughen Aluminum coatings or distort substrates

- it is desirable that, if possible, the coatings be removable and replaceable, if necessary, without damage to the underlying substrates. Ion beam polishing may allow this to be done, and should be further investigated

- full-scale IFE final optics can be fabricated and coated much more economically if they can be made in segments

While the promise of the DLC coatings originally proposed is yet to be realized, they may prove suitable with further development efforts. A parallel effort should also be initiated using the equipment design guidelines in this Report, to design an advanced, ultraclean IFE optics coating system which would produce high-quality Aluminum coatings with low-absorption oxide protective layers, by some combination of plasma deposition, ion beam-assisted deposition, sputtering, and/or evaporation processes.

Phase II and Phase III Plans. The ultimate Program goal is providing a turn-key coating process and hardware for producing long-lifetime overcoated reflective coatings for IFE final optical components in Phase III. Toward this goal, the Phase II program activities will include:

$\underline{\text { Phase II Program Activities }}$

- $\quad$ Coating Optimization

- Coating Testing and Characterization

- $\quad$ Construction of Prototype Deposition System

- $\quad$ Coating Application to Mid-Sized Substrates

- Contamination Control Engineering

- $\quad$ Engineering of Process Scaleup to Large Substrates 
- $\quad$ Finalization of Process and Equipment Specifications

- Design of Phase III Pilot Production Deposition System

- Commercial Applications Development

Phase II technical objectives include:

- Implementation of IBAD and/or sputtered protected Aluminum reflective coating process in a specially designed Phase II prototype deposition system

- Development of protective overcoatings with total optical losses (absorption and scattering) of no more than 1000 parts per million

- Coatings should withstand at least 1000 twenty nanosecond KrF laser pulses at a fluence of $3-5 \mathrm{~J} / \mathrm{cm}^{2}$

- Abrasion resistance should be consistent with existing MIL-specs for coated optics (eraser rub tests per MIL-M-13508C and MIL-E-123397).

- Deposition of protective coatings on 4-6" diameter substrates (prototypes of IFE optical components)

- Reduction of coating maintenance time and expense by at least a factor of three

- Design and specification of Phase III pilot production deposition system

The best coatings investigated in Phase I will be applied in Phase II to mid-size prototype and actual IFE optical components for detailed testing. Coating process specifications and pilot production deposition equipment will be produced for the most successful coating process. PVD Products will scale up the most successful and costeffective coating concept, by designing, constructing, and operating a prototype deposition system to apply the coatings to prototype optical components. In Phase III, full-scale production coating equipment will be made available to DoE IFE optics contractors, and commercial applications will be pursued.

If the Phase II program is successful, the potential will be demonstrated for packaging critical coated IFE optics with robust, stable, contamination-resistant coatings will be accomplished. The most successful protective coatings will have been applied to prototype and actual IFE optics and qualified for use in future IFE reactors. A concept for ultraclean deposition process and equipment will have been defined, and specifications developed for an effective IFE optical packaging coating and the equipment to make it. 
If the objectives of the Phase II program are achieved, potential commercial applications of such durable, low-loss protective coatings are numerous, including application to mirrors, windows, and lenses for lasers and laser systems of all types, as well as photographic and telescope optics, and the optical components of measurement systems and analytical instrumentation. The availability of robust packaging methods for coated optical components will have benefits in many areas of application for lasers, including material processing systems, LIDAR systems, and aerospace systems. Many other applications will be served in data storage, wear and friction reducing coatings, microelectromechanical (MEMS) devices, microelectronics manufacture, displays, and ophthalmics. If the coatings can be applied cheaply enough, then large-area commercial applications will be cost-effective, including architectural glass panels, display screens, and windows for automobiles, trains, and airplanes.

Major milestones of the Phase II program include:

- Kickoff Meeting

- Design of the Phase II prototype coating system

- Construction of the Phase II prototype coating system

- Coating and delivery of prototype SiC IFE final optical components

- Design of the Phase III pilot production coating system

- Finalization of process and equipment specifications

Building upon the results of the Phase I program and final optics development and characterization activities being done under the direction of Dr. Mark Tillack at UCSD, the most successful type of IFE final optics coating will be further optimized in the early stages of the Phase II program, with respect to optical performance, uniformity, stress, deposition rate, cleanability, and removability/reapplication. A series of test coatings will be made, and coated substrates will be tested in more detail than was possible within the budget and time frame of the Phase I program.

At this time, it is anticipated that the initial prototype optics to be coated in Phase II will consist of smooth, relatively economical 4-6" diameter glass plates and Silicon wafers. If these preliminary coatings prove acceptable, then actual mid-size superpolished 4" diameter superpolished SiC substrates will be coated, tested, and supplied to the DoE and/or UCSD. Actual IFE optical components in the same size range (if provided by the Government and/or IFE optics contractors) will then be coated at PVD Products and supplied to the cognizant laboratories for testing for resistance to IFE environmental effects. 
Design and Construction of Prototype Deposition System. In Phase II, a dedicated coating system will be designed and constructed at PVD Products, which will be capable of depositing the downselected Phase I coating(s) over an area 6" $(15 \mathrm{~cm})$ in diameter, representative of commercially available $\mathrm{SiC}$ substrates. Based on the lessons learned with this mid-size Phase II coating system, a design, specification, and cost estimate will be prepared for a large Phase III coating system capable of coating larger segments and full-size final optics.

The most successful coating process will be downselected and scaled up, in two discrete stages:

- a prototype deposition system will be designed and constructed in Phase II, which will be capable of coating substrates within an area approximately 6 " in diameter. The system will incorporate contamination monitoring by residual gas analysis, as well as optical monitoring of coating properties, and substrate front surface temperature. An ion source will be employed for substrate precleaning, and to bombard the substrates simultaneously with condensation of the coating material atoms on the surface. The system will also be used for testing whether deposited protective coatings can be safely removed by ion beam polishing/etching, and then re-applied. Phase III systems may require long linear ion sources to cover large substrates.

- based on experience with the prototype system, a full-scale deposition system capable of coating substrates of the appropriate size for IFE final optics (up to 1 meter) will be designed, and a specification and cost estimate prepared for the Phase II Final Report. This system may be designed as an in-line system with entry and exit load-locks and a conveyor belt substrate transportation system, or as a cluster tool with a substrate manipulator and central entry/exit chamber, separated by isolation valves from separate chambers for each stage of the process.

Coating Deposition, Characterization, and Optimization. A coating uniformity test fixture will be designed and constructed, and employed in the Phase II prototype deposition system to allow the modeling of the expected uniformity to be achieved when and if large (up to 1 meter long by 1/4 meter wide) substrates are coated in a full-scale deposition system, to be constructed in Phase III.

To learn as much as possible from a limited set of trials and samples, the experimental protocol will include pre-deposition masking of selected samples, to provide a pristine reference area alongside the coated area to facilitate characterization and analysis of the coating's properties. 
Process optimization test coatings will first be subjected to a brief series of screening tests for adherence, durability, and optical transmission. Coatings that pass the screening tests will then be evaluated more thoroughly, for microstructure, stress, laser damage resistance, refractive index, and optical losses due to both scattering and absorption. Mechanical properties including stress, abrasion resistance, and adherence will be quantitatively measured. Coating impurities and interfacial contamination will be measured in detail, and correlated with measured absorption losses, and minimized by tracing them back to their sources in the process and equipment.

Coating Application to 4" Substrates. The prototype deposition system will be used to coat larger substrates than the Phase I system was capable of. The substrate configurations, materials, and optical specifications will be chosen in consultation with the DoE Technical Monitor. A limited number of coated 4" substrates will be supplied to DoE and/or their designated contractors for testing.

Because the cost of actual IFE final optics components is beyond the scope of a Phase II SBIR program, preliminary test coatings in this Task will be done first on arrays of several premasked, inexpensive glass and Silicon substrates, positioned in a 6" diameter uniformity test fixture. When good uniformity has been obtained, coatings will then be done on superpolished $\mathrm{SiC}$ substrates supplied by Rohm and Haas, initially $30 \mathrm{~mm}$ diameter, followed by 4" diameter. If these coatings are successful, any actual final optics substrates supplied by the DoE and/or its contractors will then be coated at PVD Products, and supplied to the appropriate laboratories for testing, as determined at the Phase II program Kickoff Meeting.

Contamination Control. In Phase II and Phase III, the deposition system design principles and SEMATECH specifications listed above will be implemented to the greatest extent technically and economically feasible, in the design and construction of deposition equipment. The expert services of Dr. John O'Hanlon, former director of the NSF Center for Microcontamination Control and longtime semiconductor industry veteran, will be invaluable in the course of this critical Task. Dr. O'Hanlon was a design consultant on the Low Defect Deposition System now being used at Lawrence Livermore National Laboratory to make extremely low-particulate contamination optical coatings for extreme UV lithography reflective masks. Mr. Stelmack, the PI on this program, was also a consultant on the same LLNL system. 
Process Scaleup Engineering, and Design of Pilot Production System. The systematic scale-up of promising low-maintenance protective coatings for IFE final optics will take place in three stages, over the course of the Phase II and Phase III programs. Issues to be addressed in Phase II process scaleup activities include:

- Coating uniformity

- Contamination control engineering

- Process economics and deposition rates

- Development of final process and equipment specifications

A pilot production system for coating IFE optical components up to one meter in length will be specified and designed in Phase II, for construction and delivery in Phase III. In order to translate the results of this program into concrete, well-documented accomplishments, detailed specifications will be drawn up for both an optimized coating process, and the required procedures and equipment. Testing procedures for the performance, and cleanability of coated IFE final optics will be developed, in consultation with the DoE Technical monitor.

Reporting and Deliverables. The results of the Phase II coating, testing, analytical, and engineering activities will be summarized, as well as the designs of the Phase II and Phase III coating systems, and a deposition process specification. Test data will be correlated with corresponding substrate treatments and process parameters, to determine the most effective method for application of IFE final optics coatings. Specifications will be developed, both for the best coating process, and the associated ultraclean deposition equipment.

A final report will be prepared, containing data summaries, conclusions of the Phase II program, and process and equipment specifications. A summary will be provided of the plans for making deposition equipment commercially available in Phase III for meeting DoE IFE optics coating requirements.

Deliverables will include a limited number of mid-size (nominally 4") coated prototype SiC final optics components, which may be evaluated as desired by DoE or its designated contractors. If substrates and shipping containers are supplied by the Government or its contractors, PVD Products will coat actual mid-size IFE optical components up to 6" in size, and will deliver the coated optics to the cognizant laboratories for testing. 
Phase II Project Team. The planned Phase II subcontractors and their activities are as follows:

$\begin{array}{ll}\text { Subcontractor } & \text { Activities } \\ \text { Rohm and Haas } & \text { SiC substrate fabrication } \\ \text { Opco Laboratory } & \begin{array}{l}\text { Glass substrate fabrication } \\ \text { Optical testing of coatings } \\ \text { Potential deposition equipment customer }\end{array} \\ \begin{array}{l}\text { Southwest Research } \\ \text { Institute }\end{array} & \begin{array}{l}\text { Coating deposition and analysis } \\ \text { Process technology transfer }\end{array} \\ \text { Optical Coating Corporation } & \begin{array}{l}\text { MIL-spec environmental tests } \\ \text { Potential deposition equipment customer }\end{array} \\ \text { Dr. John O'Hanlon } & \text { Contamination control engineering } \\ \text { Geller Analytical } & \text { Compositional analysis }\end{array}$

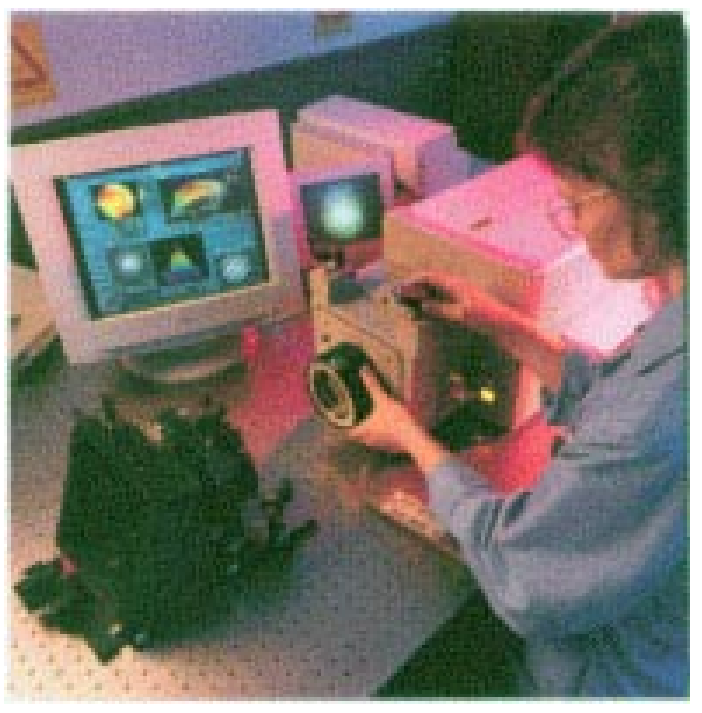

Figure 43. Optical Surface Testing at OPCO Laboratory.

In addition, the University of California at San Diego will carry out testing and analysis of coating samples, as part of the ARIES program, under the direction of Dr. Mark Tillack. 


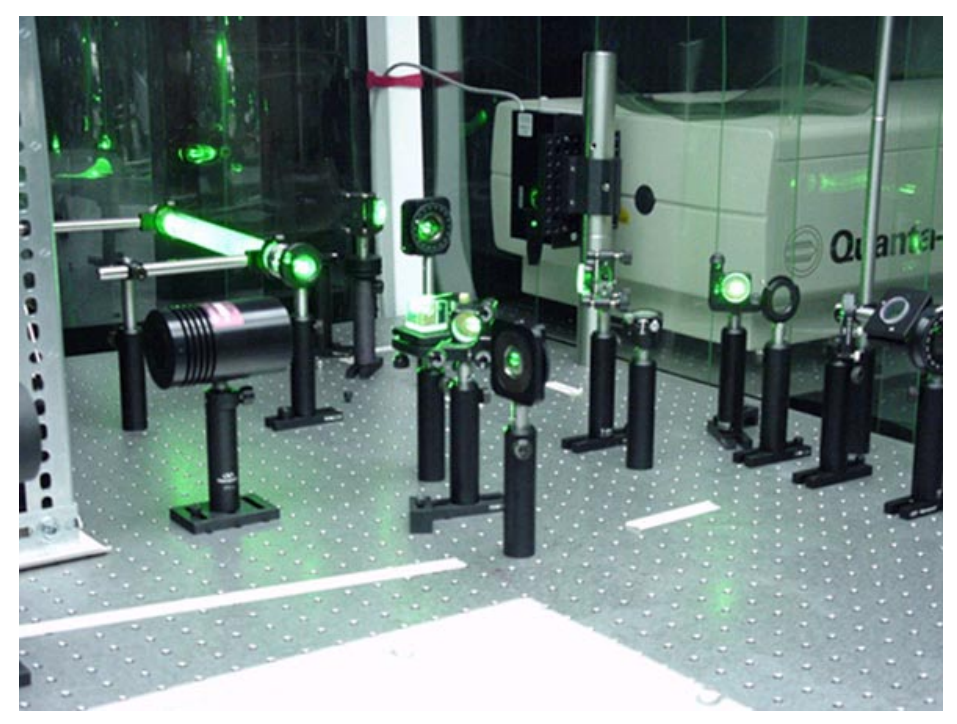

Figure 44. Laser Damage Test Facility at UC San Diego.

Phase III Activities. The coating process optimized in the early stages of Phase II, will be scaled up to meet the government's needs to coat full-scale IFE optical components in the design of the Phase III production coating system. PVD Products will ultimately provide to the DoE and/or its contractors in Phase III a commercially available turn-key ultra-clean coating process and hardware for producing long-lifetime protected reflective coatings for IFE final optical components. Commercial applications will also be pursued. 


\section{$\underline{\text { References }}$}

1. "Contamination and optics degradation as related to an evolving mission design for the Terrestrial Planet Finder", P. K. Sharma and C. A. Lindensmith (JPL), AAAS Meeting \#193, Paper 12.05 (1/6/99)

2. "Optical characteristics of Teflon AF fluoroplastic materials", J. H. Lowry et al, Opt. Eng. 31(9) 1982 (9/92).

3. "Contact angle, wetting, and adhesion: a critical review", R. J. Good, p. 3-36 in Contact Angle, Wettability, and Adhesion, ed. K. L. Mittal, VSP Publisher, 1993.

4. "Application-oriented modifications of deposition processes for diamond-like-carbonbased coating", M. Grischke et al, Surf. Coat. Technol. 74-75 739 (1995).

5. "Ion beam deposition of fluorinated amorphous carbon", C. Ronning et al (U. Gottingen), J. Appl. Phys. 90(8) 4237 (10/15/01).

6. "Fluorinated DLC for Magnetic Recording Devices", US Patent no. 5,674,638, A. Grill and V. V. Patel, IBM Corp. (10/2/95).

7. "Optical properties of fluorinated diamond-like carbon films produced by pulsed glow discharge plasma immersion ion plating", M. Hakovirta et al (M. Nastasi group at LANL), J. Appl. Phys. $\underline{\text { 88(3) }} 1456$ (8/1/00).

8. "Amorphous carbon coatings prepared by high rate if plasma decomposition from fluorinated benzenes", R. E. Sah et al, Appl. Phys. Lett. 46(8) 739 (4/15/85).

9. "Production and wetting properties of fluorinated diamond-like carbon coatings", R. S. Butter et al, Thin Solid Films $\underline{311} 107$ (1997).

10. "Ion Beam Assisted Thin Film Deposition", J. K. Hirvonen, Mater. Sci. Report 6 (6), July 1991.

11. "Ion Beam Processing for Surface Modification", J. K. Hirvonen, Ann. Rev. Mater. Sci. 19401 (1989).

12. "Plasma Immersion Ion Implantation", J. V. Mantese et al, MRS Bulletin, Aug. 1996, p. 52.

13. "Surface modification by plasma immersion ion processing", K. C. Walter et al, Proc. SPIE $\underline{3413}, 156$ (Sept. 1998).

14. "Adherent diamond-like carbon coatings on metals via plasma source ion implantation", K. C. Walter et al, Surf. Coat. Technol. 93(2-3) 287 (1997). 
15. "Barrier properties of carbon films deposited on polymer-based devices in aggressive environments", V. M. Elinson et al, Diamond Rel. Mater. 8(12) 2103 (1999).

16. "Radiation hardness of DLC films produced by ion-assisted deposition", S. Prawer et al, Diamond Rel. Mater. 5(3-5) 405 (1996).

17. "Study of diamond-like carbon films for protective coatings", V. Kh. Kudoyarova et al, Surf. Coat. Technol. 100-101(1-3) 192 (1998).

18. "Diamond-like carbon thin films as antireflective and protective coatings of GaAs elements and devices", L. Klibanov et al, Opt. Eng. 39(4) 989 (2000).

19. "Metal oxynitride and diamond hard coatings for infrared windows", A. J. Miller et al, Proc. SPIE 3705142 (1999).

20. "Fabrication and testing of diamond coatings on infrared windows for the Harrier GR7 and AV8-B systems", M. D. Hudson et al, Proc. SPIE $\underline{3060} 196$ (1997).

21. "Diamond coatings for large-area IR windows", C. J. Brierley et al, Proc. SPIE $\underline{2286}$ 307 (1994).

22. "Application of diamond-like carbon film as protection and antireflection coating of ZnS elements", G. Zhang et al, Opt. Eng. 33(4) 1330 (1994).

23. "Diamond-like carbon protective coatings for optical windows", D. M. Swec and M. J. Mirtich, Proc. SPIE 1112162 (1989).

24. "Deposition and laser damage tests of DLC coatings on silica optical fibers and plates", S. V. Garnov et al, Proc. SPIE 34849 (1998).

25. "High-durability infrared transparent coatings", L. M. Goldman and R. W. Tustison, Proc. SPIE 2286316 (1994).

26. "Sand impact testing of durable coating on FLIR ZnS relevant to the LANTIRN E-O system window", E. M. Waddell et al, Proc. SPIE 2286364 (1994).

27. "Broadband IR transparent rain and sand erosion protective coating for the F14 aircraft infrared search and track germanium dome", E. M. Waddell et al, Proc. SPIE $\underline{2286} 376$ (1994).

28. "Diamond layers for the protection of infrared windows", D. A. Tossell et al, Proc. SPIE 1760268 (1992).

29. "Optical and other properties of MPACVD diamond", C. J. H. Wort et al, Proc. SPIE $\underline{1112} 199$ (1989). 
30. "IR uses of diamond and diamond-like thin films", A. H. Lettington, Proc. SPIE 1112 384 (1989).

31. "Diamond-coated $\mathrm{ZnS}$ for improved erosion resistance", R. Korenstein et al, Proc. SPIE $\underline{3060} 181$ (1997).

32. "Diamond-like carbon films in multilayered interference coatings for IR optical elements", O. M. Kutsay et al, Diamond Rel. Mater. 10 (9-10) 1846 (2001).

33. "Protective diamond-like coatings for optical materials and electronic devices", N. V. Novikov et al, Diamond Rel. Mater. 9(3-6) 792 (2000).

34. "Optical transmittance of antireflective diamond-like coatings on ZnS substrates", G. F. Zhang and X. Zheng, Surf. Coat. Technol. 82(1-2) 110 (1996).

35. "Properties of GaAs solar cells coated with diamondlike carbon films", M. AloneAlaluf $e t$ al, Thin Solid Films $\underline{\text { 320(2) }} 159$ (1998).

36. "Application of diamond-like carbon films as hermetic coatings on optical fibres", D. P. Dowling et al, Diamond Rel. Mater. 5(3-5) 492 (1996).

37. "Amorphous diamond-like carbon films - A hard anti-reflecting coating for silicon solar cells", M. Alaluf et al, Thin Solid Films 256(1-2) 1 (1995).

38. "Tribological properties, stoichiometry and surface roughness of Si-DLC IBAD

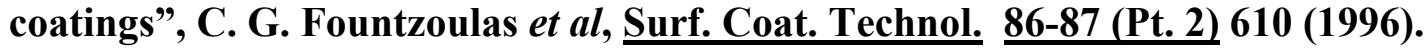

39. "Increased adhesion of diamond-like carbon-Si coatings and its tribological properties", H. Mori and H. Tachikawa, Surf. Coat. Technol. 149(2-3) 224 (2002).

40. "Comparative study of the tribological moisture sensitivity of Si-free and Si-containing diamond-like carbon films", R. Gilmore and R. Hauert, Surf. Coat. Technol. 133-134 437 (2000).

41. "Wear behavior of silicon-containing diamond-like carbon coatings", W. -J. Wu et al, Diamond Rel. Mater. 7(10) 1478 (1998).

42. "Variation of the wettability of DLC-coatings by network modification using silicon and oxygen", M. Grischke et al, Diamond Rel. Mater. 7(2-5) 454 (1998).

43. "Improvement of the efficiency of the silicon solar cells by silicon incorporated diamond-like carbon antireflective coatings", V. Bursikova et al, J. Non-Cryst. Solids 299302(Pt. 2) 1147 (2002).

\section{Plasma Immersion Ion Processing}


"Handbook of Plasma Immersion Ion Implantation and Deposition," A. Anders, John Wiley publishers, 2000.

J. R. Conrad, US Patent No. 4,764,394, "Method and apparatus for plasma source ion implantation", (August 1988).

G. W. Malaczynski et al, US Patent No. 5, 458, 927, "Process for the formation of wearand scuff-resistant carbon coatings", (October 1995).

\section{General References on Diamondlike Carbon Protective Optical Coatings}

References on DLC coatings and their relevant optical and protective applications not listed above are given below, as part of the literature search conducted in the course of this program. The assistance of Dr. James K. Hirvonen of the Army Research Laboratory in compiling these references is gratefully acknowledged.

\section{GENERAL}

"Update on diamond and diamond-like carbon coatings", A. H. Lettington, Proc. SPIE $\underline{1320} 406$ (1990).

"Diamond-like carbon thin films prepared by rf-plasma CVD", J. Jiang and C. Z. Lu, Proc. SPIE 1519717 (1991).

"Preparation of hydrophobic diamond like carbon films by plasma source ion implantation", R. Hatada and K. Baba, Nucl. Insts. Meth. Phys. Res. B148(1-4) 655 (1999).

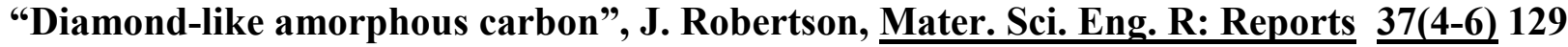
(2002).

"Improvement of the adhesion of diamond-like carbon coatings induced by ion treatments", Der-Jun Jan and Chi-Fong Ai, Materials Chemistry and Physics $72(2) 158$ (2001).

"Use of low-energy ion assistance for the modification of diamond-like carbon films

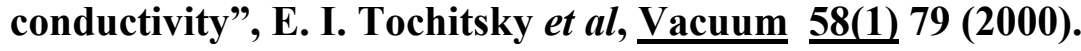

"Ion beam assisted deposition of diamond-like nanocomposite films in an acetylene atmosphere", X. -Z. Ding et al, Thin Solid Films $\underline{346(1-2)} 82$ (1999). 
"Diamond-like carbon films with extremely low stress", R. Bhattacharyya et al, Thin Solid $\underline{\text { Films }} \underline{\text { 346(1-2) }} 130$ (1999).

"A study of microstructural and electrochemical properties of ultra-thin DLC coatings on AITiC substrates deposited using the ion beam technique", Z. H. Liu et al, Diamond Rel. Mater. 8(1) 56 (1999).

"Adherent diamond-like carbon coatings on metals via plasma source ion implantation", K. C. Walter et al, Surf. Coat. Technol. 93(2-3) 287 (1997).

\section{OPTICAL APPLICATIONS}

"The effect of refractive index on the friction coefficient of DLC coated polymer substrates", K. Donnelly et al, Diamond Rel. Mater. 8(2-5) 538 (1999).

"Normal and interfacial stresses in thin film coated optics: the case of diamond-coated zinc-sulfide windows", C. A. Klein, Proc. SPIE $\underline{4198} 122$ (2001).

"Frontiers in infrared window and dome materials", D. C. Harris, Proc. SPIE 2552325 (1995).

"Rain erosion data on window and dome materials", C. R. Seward et al, Proc. SPIE 1760 280 (1992).

"Technology trends for high performance windows", J. Askinazi, in Tactical Infrared Systems: Proceedings, Proc. SPIE 1498100 (1991).

"The influence of diamond-like carbon films on the properties of silicon solar cells", M. Allon-Alaluf et al, Thin Solid Films $\underline{\text { 303(1-2) }} 273$ (1997).

"Use of diamond-like carbon films in X-ray optics", P. E. Kondrashov et al, Diamond Rel. Mater. 6(5-7) 902 (1997). 